August 14, 2021

J. Phys. Chem. A

Revised

\title{
Supplementary Materials for \\ A Microwave Study of Triflic Acid Hydrates: Evidence for the Transition from Hydrogen Bonded Clusters to a Microsolvated Ion Pair
}

\author{
Anna K. Huff, Nathan Love, and Kenneth R. Leopold* \\ Department of Chemistry \\ University of Minnesota \\ 207 Pleasant St., SE \\ Minneapolis, MN 55455
}

* Corresponding Author: Email kleopold@umn.edu 
Table S1. Observed transitions for $\mathrm{CF}_{3} \mathrm{SO}_{3} \mathrm{H} \cdots \mathrm{H}_{2} \mathrm{O}$

\begin{tabular}{|c|c|c|c|c|c|c|c|c|}
\hline$J^{\prime}$ & $K_{a}$ & $K_{c}^{\prime}$ & $J^{\prime \prime}$ & $K_{a} "$ & $K_{c}{ }^{\prime \prime}$ & State & $\begin{array}{c}\text { Observed Frequency } \\
(\mathrm{MHz})\end{array}$ & $\begin{array}{c}\text { Obs-Calc } \\
(\mathrm{kHz})\end{array}$ \\
\hline 2 & 1 & 2 & 1 & 1 & 1 & $0^{-}$ & 4023.479 & 0.000 \\
\hline 2 & 1 & 2 & 1 & 1 & 1 & $0^{+}$ & 4023.563 & 0.001 \\
\hline 2 & 0 & 2 & 1 & 0 & 1 & $0^{-}$ & 4176.940 & 0.001 \\
\hline 2 & 0 & 2 & 1 & 0 & 1 & $0^{+}$ & 4177.016 & 0.000 \\
\hline 2 & 1 & 1 & 1 & 1 & 0 & $0^{-}$ & 4394.102 & 0.001 \\
\hline 2 & 1 & 1 & 1 & 1 & 0 & $0^{+}$ & 4394.156 & -0.004 \\
\hline 2 & 1 & 2 & 1 & 0 & 1 & $0^{-}$ & 4731.731 & 0.000 \\
\hline 2 & 1 & 2 & 1 & 0 & 1 & $0^{+}$ & 4731.856 & 0.000 \\
\hline 3 & 0 & 3 & 2 & 1 & 2 & $0^{-}$ & 5636.754 & 0.000 \\
\hline 3 & 0 & 3 & 2 & 1 & 2 & $0^{+}$ & 5636.833 & 0.001 \\
\hline 3 & 1 & 3 & 2 & 1 & 2 & $0^{-}$ & 6016.750 & 0.000 \\
\hline 3 & 1 & 3 & 2 & 1 & 2 & $0^{+}$ & 6016.878 & 0.000 \\
\hline 3 & 0 & 3 & 2 & 0 & 2 & $0^{-}$ & 6191.545 & 0.000 \\
\hline 3 & 0 & 3 & 2 & 0 & 2 & $0^{+}$ & 6191.672 & 0.000 \\
\hline 3 & 2 & 2 & 2 & 2 & 1 & $0^{-}$ & 6313.190 & 0.000 \\
\hline 3 & 2 & 2 & 2 & 2 & 1 & $0^{+}$ & 6313.296 & 0.000 \\
\hline 3 & 2 & 1 & 2 & 2 & 0 & $0^{-}$ & 6434.777 & -0.001 \\
\hline 3 & 2 & 1 & 2 & 2 & 0 & $0^{+}$ & 6434.863 & 0.000 \\
\hline 2 & 2 & 1 & 1 & 1 & 0 & $0^{-}$ & 6518.849 & 0.002 \\
\hline 2 & 2 & 1 & 1 & 1 & 0 & $0^{+}$ & 6519.034 & 0.002 \\
\hline 3 & 1 & 2 & 2 & 1 & 1 & $0^{-}$ & 6569.177 & 0.000 \\
\hline 3 & 1 & 2 & 2 & 1 & 1 & $0^{+}$ & 6569.268 & -0.001 \\
\hline 3 & 1 & 3 & 2 & 0 & 2 & $0^{-}$ & 6571.540 & -0.001 \\
\hline 3 & 1 & 3 & 2 & 0 & 2 & $0^{+}$ & 6571.719 & 0.001 \\
\hline 2 & 2 & 0 & 1 & 1 & 1 & $0^{-}$ & 6736.005 & 0.000 \\
\hline 2 & 2 & 0 & 1 & 1 & 1 & $0^{+}$ & 6736.174 & 0.003 \\
\hline 4 & 0 & 4 & 3 & 1 & 3 & $0^{-}$ & 7763.246 & 0.000 \\
\hline 4 & 0 & 4 & 3 & 1 & 3 & $0^{+}$ & 7763.378 & 0.000 \\
\hline 4 & 1 & 4 & 3 & 1 & 3 & $0^{-}$ & 7992.310 & -0.002 \\
\hline 4 & 1 & 4 & 3 & 1 & 3 & $0^{+}$ & 7992.488 & 0.001 \\
\hline 4 & 0 & 4 & 3 & 0 & 3 & $0^{-}$ & 8143.241 & -0.001 \\
\hline 4 & 0 & 4 & 3 & 0 & 3 & $0^{+}$ & 8143.425 & 0.000 \\
\hline 4 & 1 & 4 & 3 & 0 & 3 & $0^{-}$ & 8372.307 & 0.000 \\
\hline 4 & 1 & 4 & 3 & 0 & 3 & $0^{+}$ & 8372.533 & 0.000 \\
\hline 4 & 2 & 3 & 3 & 2 & 2 & $0^{-}$ & 8392.576 & 0.001 \\
\hline 4 & 2 & 3 & 3 & 2 & 2 & $0^{+}$ & 8392.720 & -0.001 \\
\hline 3 & 2 & 2 & 2 & 1 & 1 & $0^{-}$ & 8437.935 & -0.001 \\
\hline 3 & 2 & 2 & 2 & 1 & 1 & $0^{+}$ & 8438.167 & -0.002 \\
\hline 4 & 3 & 2 & 3 & 3 & 1 & $0^{-}$ & 8472.180 & 0.002 \\
\hline 4 & 3 & 2 & 3 & 3 & 1 & $0^{+}$ & 8472.310 & 0.000 \\
\hline
\end{tabular}


Table S1. Observed transitions for $\mathrm{CF}_{3} \mathrm{SO}_{3} \mathrm{H} \cdots \mathrm{H}_{2} \mathrm{O}$ (continued)

\begin{tabular}{|c|c|c|c|c|c|c|c|c|}
\hline$J^{\prime}$ & $K_{a}$ & $K_{c}^{\prime}$ & $J^{\prime \prime}$ & $K_{a} ”$ & $K_{c} "$ & State & $\begin{array}{c}\text { Observed Frequency } \\
(\mathrm{MHz})\end{array}$ & $\begin{array}{c}\text { Obs-Calc } \\
(\mathrm{kHz})\end{array}$ \\
\hline 4 & 3 & 1 & 3 & 3 & 0 & $0^{-}$ & 8492.638 & 0.000 \\
\hline 4 & 3 & 1 & 3 & 3 & 0 & $0^{+}$ & 8492.768 & 0.003 \\
\hline 4 & 2 & 2 & 3 & 2 & 1 & $0^{-}$ & 8666.458 & 0.000 \\
\hline 4 & 2 & 2 & 3 & 2 & 1 & $0^{+}$ & 8666.563 & 0.000 \\
\hline 4 & 1 & 3 & 3 & 1 & 2 & $0^{-}$ & 8713.014 & 0.000 \\
\hline 4 & 1 & 3 & 3 & 1 & 2 & $0^{+}$ & 8713.146 & 0.000 \\
\hline 3 & 2 & 1 & 2 & 1 & 2 & $0^{-}$ & 9147.305 & 0.001 \\
\hline 3 & 2 & 1 & 2 & 1 & 2 & $0^{+}$ & 9147.466 & -0.007 \\
\hline 5 & 1 & 4 & 4 & 2 & 3 & $0^{-}$ & 9259.926 & -0.002 \\
\hline 5 & 0 & 5 & 4 & 1 & 4 & $0^{-}$ & 9825.862 & 0.001 \\
\hline 5 & 0 & 5 & 4 & 1 & 4 & $0^{+}$ & 9826.053 & -0.002 \\
\hline 5 & 1 & 5 & 4 & 1 & 4 & $0^{-}$ & 9950.381 & 0.000 \\
\hline 5 & 1 & 5 & 4 & 1 & 4 & $0^{+}$ & 9950.606 & 0.001 \\
\hline 5 & 0 & 5 & 4 & 0 & 4 & $0^{-}$ & 10054.927 & 0.000 \\
\hline 5 & 0 & 5 & 4 & 0 & 4 & $0^{+}$ & 10055.163 & 0.000 \\
\hline 5 & 1 & 5 & 4 & 0 & 4 & $0^{-}$ & 10179.447 & 0.001 \\
\hline 5 & 1 & 5 & 4 & 0 & 4 & $0^{+}$ & 10179.714 & 0.000 \\
\hline 4 & 2 & 3 & 3 & 1 & 2 & $0^{-}$ & 10261.333 & 0.000 \\
\hline 4 & 2 & 3 & 3 & 1 & 2 & $0^{+}$ & 10261.620 & -0.001 \\
\hline 3 & 3 & 1 & 2 & 2 & 0 & $0^{-}$ & 10304.233 & -0.011 \\
\hline 3 & 3 & 0 & 2 & 2 & 1 & $0^{-}$ & 10339.557 & -0.009 \\
\hline 5 & 2 & 4 & 4 & 2 & 3 & $0^{-}$ & 10451.231 & 0.000 \\
\hline 5 & 2 & 4 & 4 & 2 & 3 & $0^{+}$ & 10451.421 & 0.001 \\
\hline 5 & 3 & 3 & 4 & 3 & 2 & $0^{-}$ & 10599.815 & 0.003 \\
\hline 5 & 3 & 3 & 4 & 3 & 2 & $0^{+}$ & 10599.973 & -0.004 \\
\hline 5 & 3 & 2 & 4 & 3 & 1 & $0^{-}$ & 10668.291 & -0.006 \\
\hline 5 & 1 & 4 & 4 & 1 & 3 & $0^{-}$ & 10808.247 & 0.000 \\
\hline 5 & 1 & 4 & 4 & 1 & 3 & $0^{+}$ & 10808.430 & 0.002 \\
\hline 5 & 2 & 3 & 4 & 2 & 2 & $0^{-}$ & 10915.507 & 0.000 \\
\hline 5 & 2 & 3 & 4 & 2 & 2 & $0^{+}$ & 10915.638 & 0.000 \\
\hline 6 & 1 & 5 & 5 & 2 & 4 & $0^{-}$ & 11646.187 & 0.009 \\
\hline 4 & 2 & 2 & 3 & 1 & 3 & $0^{-}$ & 11797.014 & 0.001 \\
\hline 4 & 2 & 2 & 3 & 1 & 3 & $0^{+}$ & 11797.159 & 0.001 \\
\hline 6 & 0 & 6 & 5 & 1 & 5 & $0^{-}$ & 11831.025 & -0.001 \\
\hline 6 & 0 & 6 & 5 & 1 & 5 & $0^{+}$ & 11831.281 & 0.001 \\
\hline 6 & 1 & 6 & 5 & 1 & 5 & $0^{-}$ & 11893.887 & 0.000 \\
\hline 6 & 1 & 6 & 5 & 1 & 5 & $0^{+}$ & 11894.160 & 0.000 \\
\hline 6 & 0 & 6 & 5 & 0 & 5 & $0^{-}$ & 11955.546 & 0.000 \\
\hline 6 & 0 & 6 & 5 & 0 & 5 & $0^{+}$ & 11955.832 & 0.001 \\
\hline 5 & 2 & 4 & 4 & 1 & 3 & $0^{-}$ & 11999.550 & 0.000 \\
\hline
\end{tabular}


Table S1. Observed transitions for $\mathrm{CF}_{3} \mathrm{SO}_{3} \mathrm{H} \cdots \mathrm{H}_{2} \mathrm{O}$ (continued)

\begin{tabular}{|c|c|c|c|c|c|c|c|c|}
\hline$J^{\prime}$ & $K_{a}$ & $K_{c}^{\prime}$ & $J^{\prime \prime}$ & $K_{a} ”$ & $K_{c} "$ & State & $\begin{array}{c}\text { Observed Frequency } \\
(\mathrm{MHz})\end{array}$ & $\begin{array}{c}\text { Obs-Calc } \\
(\mathrm{kHz})\end{array}$ \\
\hline 5 & 2 & 4 & 4 & 1 & 3 & $0^{+}$ & 11999.895 & 0.000 \\
\hline 6 & 1 & 6 & 5 & 0 & 5 & $0^{-}$ & 12018.407 & 0.001 \\
\hline 6 & 1 & 6 & 5 & 0 & 5 & $0^{+}$ & 12018.711 & 0.000 \\
\hline 4 & 3 & 2 & 3 & 2 & 1 & $0^{-}$ & 12341.634 & -0.010 \\
\hline 7 & 2 & 5 & 6 & 3 & 4 & $0^{-}$ & 12393.395 & -0.018 \\
\hline 6 & 2 & 5 & 5 & 2 & 4 & $0^{-}$ & 12485.636 & -0.009 \\
\hline 6 & 2 & 5 & 5 & 2 & 4 & $0^{+}$ & 12485.878 & -0.003 \\
\hline 4 & 3 & 1 & 3 & 2 & 2 & $0^{-}$ & 12519.008 & -0.006 \\
\hline 4 & 3 & 1 & 3 & 2 & 2 & $0^{+}$ & 12519.328 & 0.011 \\
\hline 6 & 3 & 4 & 5 & 3 & 3 & $0^{-}$ & 12721.790 & -0.001 \\
\hline 6 & 3 & 4 & 5 & 3 & 3 & $0^{+}$ & 12721.997 & 0.004 \\
\hline 6 & 1 & 5 & 5 & 1 & 4 & $0^{-}$ & 12837.481 & 0.000 \\
\hline 6 & 1 & 5 & 5 & 1 & 4 & $0^{+}$ & 12837.724 & 0.000 \\
\hline 6 & 3 & 3 & 5 & 3 & 2 & $0^{-}$ & 12889.476 & -0.007 \\
\hline 6 & 3 & 3 & 5 & 3 & 2 & $0^{+}$ & 12889.635 & -0.008 \\
\hline 6 & 2 & 4 & 5 & 2 & 3 & $0^{-}$ & 13145.036 & -0.006 \\
\hline 6 & 2 & 4 & 5 & 2 & 3 & $0^{+}$ & 13145.211 & 0.001 \\
\hline 6 & 2 & 5 & 5 & 1 & 4 & $0^{-}$ & 13676.947 & -0.001 \\
\hline 6 & 2 & 5 & 5 & 1 & 4 & $0^{+}$ & 13677.338 & -0.011 \\
\hline 7 & 0 & 7 & 6 & 1 & 6 & $0^{-}$ & 13796.753 & -0.005 \\
\hline 7 & 0 & 7 & 6 & 1 & 6 & $0^{+}$ & 13797.070 & 0.000 \\
\hline 7 & 1 & 7 & 6 & 1 & 6 & $0^{-}$ & 13826.914 & -0.001 \\
\hline 7 & 1 & 7 & 6 & 1 & 6 & $0^{+}$ & 13827.237 & 0.000 \\
\hline 7 & 0 & 7 & 6 & 0 & 6 & $0^{-}$ & 13859.615 & -0.004 \\
\hline 7 & 0 & 7 & 6 & 0 & 6 & $0^{+}$ & 13859.950 & 0.000 \\
\hline 7 & 1 & 7 & 6 & 0 & 6 & $0^{-}$ & 13889.771 & -0.004 \\
\hline 7 & 1 & 6 & 6 & 2 & 5 & $0^{-}$ & 13955.952 & -0.008 \\
\hline 5 & 3 & 3 & 4 & 2 & 2 & $0^{-}$ & 14274.994 & -0.003 \\
\hline 5 & 3 & 3 & 4 & 2 & 2 & $0^{+}$ & 14275.389 & -0.006 \\
\hline 7 & 2 & 6 & 6 & 2 & 5 & $0^{-}$ & 14494.270 & -0.007 \\
\hline 7 & 2 & 6 & 6 & 2 & 5 & $0^{+}$ & 14494.560 & -0.003 \\
\hline 5 & 3 & 2 & 4 & 2 & 3 & $0^{-}$ & 14794.738 & 0.001 \\
\hline 5 & 3 & 2 & 4 & 2 & 3 & $0^{+}$ & 14795.043 & 0.002 \\
\hline 7 & 1 & 6 & 6 & 1 & 5 & $0^{-}$ & 14795.430 & 0.002 \\
\hline 7 & 1 & 6 & 6 & 1 & 5 & $0^{+}$ & 14795.738 & 0.000 \\
\hline 7 & 3 & 5 & 6 & 3 & 4 & $0^{-}$ & 14830.189 & -0.001 \\
\hline 7 & 3 & 5 & 6 & 3 & 4 & $0^{+}$ & 14830.436 & 0.003 \\
\hline 7 & 4 & 3 & 6 & 4 & 2 & $0^{-}$ & 14910.871 & -0.013 \\
\hline 7 & 3 & 4 & 6 & 3 & 3 & $0^{-}$ & 15156.936 & -0.006 \\
\hline 7 & 3 & 4 & 6 & 3 & 3 & $0^{+}$ & 15157.108 & -0.005 \\
\hline
\end{tabular}


Table S1. Observed transitions for $\mathrm{CF}_{3} \mathrm{SO}_{3} \mathrm{H} \cdots \mathrm{H}_{2} \mathrm{O}$ (continued)

\begin{tabular}{|c|c|c|c|c|c|c|c|c|}
\hline$J^{\prime}$ & $K_{a}$ & $K_{c}{ }^{\prime}$ & $J^{\prime \prime}$ & $K_{a} ”$ & $K_{c}{ }^{\prime \prime}$ & State & $\begin{array}{c}\text { Observed Frequency } \\
(\mathrm{MHz})\end{array}$ & $\begin{array}{c}\text { Obs-Calc } \\
(\mathrm{kHz})\end{array}$ \\
\hline 7 & 2 & 5 & 6 & 2 & 4 & $0^{-}$ & 15329.650 & -0.003 \\
\hline 7 & 2 & 5 & 6 & 2 & 4 & $0^{+}$ & 15329.860 & -0.011 \\
\hline 7 & 2 & 6 & 6 & 1 & 5 & $0^{-}$ & 15333.739 & -0.005 \\
\hline 8 & 0 & 8 & 7 & 1 & 7 & $0^{-}$ & 15739.318 & -0.008 \\
\hline 8 & 0 & 8 & 7 & 1 & 7 & $0^{+}$ & 15739.691 & 0.000 \\
\hline 8 & 1 & 8 & 7 & 1 & 7 & $0^{-}$ & 15753.272 & -0.009 \\
\hline 8 & 1 & 8 & 7 & 1 & 7 & $0^{+}$ & 15753.652 & 0.001 \\
\hline 8 & 0 & 8 & 7 & 0 & 7 & $0^{-}$ & 15769.481 & -0.001 \\
\hline 8 & 0 & 8 & 7 & 0 & 7 & $0^{+}$ & 15769.857 & -0.001 \\
\hline 8 & 1 & 8 & 7 & 0 & 7 & $0^{-}$ & 15783.429 & -0.008 \\
\hline 8 & 1 & 8 & 7 & 0 & 7 & $0^{+}$ & 15783.822 & 0.003 \\
\hline 6 & 3 & 4 & 5 & 2 & 3 & $0^{-}$ & 16081.282 & 0.000 \\
\hline 6 & 3 & 4 & 5 & 2 & 3 & $0^{+}$ & 16081.748 & -0.002 \\
\hline 5 & 4 & 1 & 4 & 3 & 2 & $0^{-}$ & 16153.422 & 0.011 \\
\hline 8 & 1 & 7 & 7 & 2 & 6 & $0^{-}$ & 16161.856 & 0.000 \\
\hline 8 & 2 & 7 & 7 & 2 & 6 & $0^{-}$ & 16478.082 & -0.006 \\
\hline 8 & 2 & 7 & 7 & 2 & 6 & $0^{+}$ & 16478.429 & 0.003 \\
\hline 8 & 1 & 7 & 7 & 1 & 6 & $0^{-}$ & 16700.168 & -0.004 \\
\hline 8 & 1 & 7 & 7 & 1 & 6 & $0^{+}$ & 16700.536 & -0.009 \\
\hline 8 & 3 & 6 & 7 & 3 & 5 & $0^{-}$ & 16917.797 & 0.002 \\
\hline 8 & 2 & 7 & 7 & 1 & 6 & $0^{-}$ & 17016.396 & -0.008 \\
\hline 8 & 4 & 4 & 7 & 4 & 3 & $0^{-}$ & 17112.598 & -0.011 \\
\hline 6 & 3 & 3 & 5 & 2 & 4 & $0^{-}$ & 17232.992 & 0.003 \\
\hline 6 & 3 & 3 & 5 & 2 & 4 & $0^{+}$ & 17233.263 & 0.000 \\
\hline 8 & 3 & 5 & 7 & 3 & 4 & $0^{-}$ & 17447.229 & 0.016 \\
\hline 8 & 2 & 6 & 7 & 2 & 5 & $0^{-}$ & 17452.543 & 0.004 \\
\hline 9 & 0 & 9 & 8 & 1 & 8 & $0^{-}$ & 17669.529 & -0.003 \\
\hline 9 & 1 & 9 & 8 & 0 & 8 & $0^{-}$ & 17689.765 & -0.010 \\
\hline
\end{tabular}


Table S2. Observed transitions for $\mathrm{CF}_{3}{ }^{34} \mathrm{SO}_{3} \mathrm{H} \cdots \mathrm{H}_{2} \mathrm{O}$

\begin{tabular}{ccccccccc}
\hline$J^{\prime}$ & $K_{a}{ }^{\prime}$ & $K_{c}{ }^{\prime}$ & $J^{\prime}$ & $K_{a}{ }^{\prime}$ & $K_{c}{ }^{\prime}$ & State & $\begin{array}{c}\text { Observed Frequency } \\
(\mathrm{MHz})\end{array}$ & $\begin{array}{c}\text { Obs-Calc } \\
(\mathrm{kHz})\end{array}$ \\
\hline 3 & 0 & 3 & 2 & 1 & 2 & $0^{-}$ & 5634.130 & 0.002 \\
3 & 0 & 3 & 2 & 1 & 2 & $0^{+}$ & 5634.212 & 0.003 \\
3 & 1 & 3 & 2 & 1 & 2 & $0^{-}$ & 6004.708 & 0.000 \\
3 & 1 & 3 & 2 & 1 & 2 & $0^{+}$ & 6004.833 & -0.004 \\
3 & 0 & 3 & 2 & 0 & 2 & $0^{-}$ & 6179.625 & 0.000 \\
3 & 0 & 3 & 2 & 0 & 2 & $0^{+}$ & 6179.753 & 0.001 \\
3 & 1 & 3 & 2 & 0 & 2 & $0^{-}$ & 6550.203 & -0.001 \\
3 & 1 & 2 & 2 & 1 & 1 & $0^{-}$ & 6563.429 & 0.000 \\
3 & 1 & 2 & 2 & 1 & 1 & $0^{+}$ & 6563.519 & -0.002 \\
2 & 2 & 0 & 1 & 1 & 1 & $0^{-}$ & 6711.385 & -0.001 \\
2 & 2 & 0 & 1 & 1 & 1 & $0^{+}$ & 6711.549 & 0.001 \\
4 & 0 & 4 & 3 & 1 & 3 & $0^{-}$ & 7754.321 & 0.001 \\
4 & 0 & 4 & 3 & 1 & 3 & $0^{+}$ & 7754.452 & -0.001 \\
4 & 1 & 4 & 3 & 1 & 3 & $0^{-}$ & 7975.426 & 0.001 \\
4 & 1 & 4 & 3 & 1 & 3 & $0^{+}$ & 7975.601 & 0.000 \\
4 & 0 & 4 & 3 & 0 & 3 & $0^{-}$ & 8124.900 & 0.001 \\
4 & 0 & 4 & 3 & 0 & 3 & $0^{+}$ & 8125.082 & 0.001 \\
4 & 1 & 4 & 3 & 0 & 3 & $0^{-}$ & 8346.004 & 0.000 \\
4 & 1 & 4 & 3 & 0 & 3 & $0^{+}$ & 8346.229 & 0.000 \\
4 & 2 & 3 & 3 & 2 & 2 & $0^{-}$ & 8380.787 & -0.001 \\
4 & 2 & 3 & 3 & 2 & 2 & $0^{+}$ & 8380.936 & 0.001 \\
3 & 2 & 2 & 2 & 1 & 1 & $0^{-}$ & 8405.228 & -0.001 \\
4 & 2 & 2 & 3 & 2 & 1 & $0^{-}$ & 8662.013 & 0.002 \\
4 & 1 & 3 & 3 & 1 & 2 & $0^{-}$ & 8703.687 & 0.001 \\
4 & 1 & 3 & 3 & 1 & 2 & $0^{+}$ & 8703.816 & 0.001 \\
5 & 0 & 5 & 4 & 1 & 4 & $0^{-}$ & 9809.460 & 0.000 \\
5 & 0 & 5 & 4 & 1 & 4 & $0^{+}$ & 9809.647 & -0.005 \\
5 & 1 & 5 & 4 & 1 & 4 & $0^{-}$ & 9928.326 & 0.000 \\
5 & 1 & 5 & 4 & 1 & 4 & $0^{+}$ & 9928.551 & 0.001 \\
5 & 0 & 5 & 4 & 0 & 4 & $0^{-}$ & 10030.565 & 0.000 \\
5 & 1 & 5 & 4 & 0 & 4 & $0^{-}$ & 10149.430 & -0.001 \\
\hline & 2 & 3 & 3 & 1 & 2 & $0^{-}$ & 10222.589 & 0.002 \\
5 & 4 & 4 & 1 & 3 & $0^{-}$ & 10793.508 & 0.000 \\
\hline
\end{tabular}


Table S3. Observed transitions for $\mathrm{CF}_{3} \mathrm{SO}_{3} \mathrm{H} \cdots \mathrm{D}_{2} \mathrm{O}$

\begin{tabular}{cccccccc}
\hline$J^{\prime}$ & $K_{a}{ }^{\prime}$ & $K_{c}{ }^{\prime}$ & $J^{\prime \prime}$ & $K_{a}{ }^{\prime}$ & $K_{c}{ }^{\prime}$ & $\begin{array}{c}\text { Observed Frequency } \\
(\mathrm{MHz})\end{array}$ & $\begin{array}{c}\text { Obs-Calc } \\
(\mathrm{kHz})\end{array}$ \\
\hline 3 & 1 & 3 & 2 & 1 & 2 & 5792.117 & -0.001 \\
3 & 0 & 3 & 2 & 0 & 2 & 5964.661 & 0.001 \\
3 & 1 & 2 & 2 & 1 & 1 & 6326.338 & 0.001 \\
4 & 0 & 4 & 3 & 1 & 3 & 7451.847 & 0.003 \\
4 & 1 & 4 & 3 & 1 & 3 & 7694.711 & -0.001 \\
4 & 0 & 4 & 3 & 0 & 3 & 7846.723 & -0.005 \\
4 & 1 & 4 & 3 & 0 & 3 & 8089.595 & -0.001 \\
4 & 1 & 3 & 3 & 1 & 2 & 8392.834 & -0.001 \\
5 & 0 & 5 & 4 & 1 & 4 & 9445.705 & -0.003 \\
5 & 1 & 5 & 4 & 1 & 4 & 9580.640 & -0.001 \\
5 & 0 & 5 & 4 & 0 & 4 & 9688.577 & 0.001 \\
5 & 1 & 5 & 4 & 0 & 4 & 9823.509 & 0.000 \\
5 & 2 & 4 & 4 & 2 & 3 & 10064.261 & -0.002 \\
5 & 1 & 4 & 4 & 1 & 3 & 10414.690 & -0.001 \\
5 & 2 & 3 & 4 & 2 & 2 & 10503.236 & -0.001 \\
6 & 1 & 6 & 5 & 1 & 5 & 11452.447 & -0.002 \\
6 & 0 & 6 & 5 & 0 & 5 & 11517.751 & 0.000 \\
6 & 1 & 5 & 5 & 1 & 4 & 12375.379 & 0.003 \\
\hline
\end{tabular}


Table S4. Observed transitions for $\mathrm{CF}_{3} \mathrm{SO}_{3} \mathrm{H} \cdots \mathrm{DOH}$

\begin{tabular}{|c|c|c|c|c|c|c|c|c|c|}
\hline$J^{\prime}$ & $K_{a}$ & $K_{c}{ }^{\prime}$ & $F^{\prime}$ & $J^{\prime \prime}$ & $K_{a} "$ & $K_{c}{ }^{\prime \prime}$ & $F^{\prime \prime}$ & $\begin{array}{c}\text { Observed Frequency } \\
(\mathrm{MHz})\end{array}$ & $\begin{array}{c}\text { Obs-Calc } \\
(\mathrm{kHz})\end{array}$ \\
\hline 3 & 0 & 3 & 2 & 2 & 1 & 2 & 1 & 5522.821 & 0.008 \\
\hline 3 & 0 & 3 & 4 & 2 & 1 & 2 & 3 & 5522.840 & 0.000 \\
\hline 3 & 0 & 3 & 3 & 2 & 1 & 2 & 2 & 5522.849 & -0.007 \\
\hline 3 & 1 & 3 & 3 & 2 & 1 & 2 & 2 & 5922.516 & 0.000 \\
\hline 3 & 1 & 3 & 2 & 2 & 1 & 2 & 1 & 5922.524 & 0.002 \\
\hline 3 & 1 & 3 & 4 & 2 & 1 & 2 & 3 & 5922.533 & -0.004 \\
\hline 3 & 0 & 3 & 3 & 2 & 0 & 2 & 2 & 6095.259 & 0.002 \\
\hline 3 & 0 & 3 & 4 & 2 & 0 & 2 & 3 & 6095.285 & 0.002 \\
\hline 3 & 1 & 2 & 3 & 2 & 1 & 1 & 2 & 6455.088 & 0.001 \\
\hline 3 & 1 & 2 & 4 & 2 & 1 & 1 & 3 & 6455.099 & -0.004 \\
\hline 3 & 1 & 2 & 2 & 2 & 1 & 1 & 1 & 6455.118 & -0.003 \\
\hline 4 & 0 & 4 & 3 & 3 & 1 & 3 & 2 & 7622.009 & -0.002 \\
\hline 4 & 0 & 4 & 5 & 3 & 1 & 3 & 4 & 7622.022 & -0.003 \\
\hline 4 & 0 & 4 & 4 & 3 & 1 & 3 & 3 & 7622.040 & 0.005 \\
\hline 4 & 1 & 4 & 4 & 3 & 1 & 3 & 3 & 7868.863 & -0.006 \\
\hline 4 & 1 & 4 & 3 & 3 & 1 & 3 & 2 & 7868.876 & 0.000 \\
\hline 4 & 1 & 4 & 5 & 3 & 1 & 3 & 4 & 7868.893 & 0.010 \\
\hline 4 & 0 & 4 & 4 & 3 & 0 & 3 & 3 & 8021.700 & 0.004 \\
\hline 4 & 0 & 4 & 5 & 3 & 0 & 3 & 4 & 8021.720 & -0.002 \\
\hline 4 & 2 & 3 & 4 & 3 & 2 & 2 & 3 & 8253.517 & 0.004 \\
\hline 4 & 2 & 3 & 5 & 3 & 2 & 2 & 4 & 8253.541 & 0.004 \\
\hline 4 & 1 & 4 & 4 & 3 & 0 & 3 & 3 & 8268.534 & 0.005 \\
\hline 4 & 1 & 4 & 5 & 3 & 0 & 3 & 4 & 8268.578 & -0.002 \\
\hline 4 & 2 & 2 & 5 & 3 & 2 & 1 & 4 & 8507.888 & 0.003 \\
\hline 4 & 2 & 2 & 4 & 3 & 2 & 1 & 3 & 8507.895 & 0.008 \\
\hline 4 & 1 & 3 & 4 & 3 & 1 & 2 & 3 & 8565.082 & 0.001 \\
\hline 4 & 1 & 3 & 5 & 3 & 1 & 2 & 4 & 8565.099 & 0.003 \\
\hline 5 & 0 & 5 & 4 & 4 & 1 & 4 & 3 & 9660.896 & 0.003 \\
\hline 5 & 0 & 5 & 6 & 4 & 1 & 4 & 5 & 9660.902 & 0.001 \\
\hline 5 & 1 & 5 & 5 & 4 & 1 & 4 & 4 & 9798.665 & 0.000 \\
\hline 5 & 1 & 5 & 4 & 4 & 1 & 4 & 3 & 9798.670 & -0.002 \\
\hline 5 & 1 & 5 & 6 & 4 & 1 & 4 & 5 & 9798.675 & -0.002 \\
\hline 5 & 0 & 5 & 5 & 4 & 0 & 4 & 4 & 9907.735 & -0.002 \\
\hline 5 & 0 & 5 & 6 & 4 & 0 & 4 & 5 & 9907.755 & -0.004 \\
\hline 5 & 1 & 5 & 5 & 4 & 0 & 4 & 4 & 10045.498 & -0.001 \\
\hline 5 & 1 & 5 & 6 & 4 & 0 & 4 & 5 & 10045.532 & -0.003 \\
\hline 3 & 3 & 1 & 4 & 2 & 2 & 0 & 3 & 10250.144 & -0.005 \\
\hline 5 & 2 & 4 & 6 & 4 & 2 & 3 & 5 & 10280.648 & -0.001 \\
\hline 3 & 3 & 0 & 4 & 2 & 2 & 1 & 3 & 10282.400 & 0.003 \\
\hline 5 & 3 & 2 & 6 & 4 & 3 & 1 & 5 & 10478.313 & -0.003 \\
\hline
\end{tabular}


Table S4. Observed transitions for $\mathrm{CF}_{3} \mathrm{SO}_{3} \mathrm{H} \cdots \mathrm{DOH}$ (continued)

\begin{tabular}{cccccccccc}
\hline$J$ & $K_{a}{ }^{\prime}$ & $K_{c}{ }^{\prime}$ & $F^{\prime}$ & $J^{\prime \prime}$ & $K_{a}{ }^{\prime}$ & $K_{c}{ }^{\prime}$ & $F ”$ & $\begin{array}{c}\text { Observed Frequency } \\
(\mathrm{MHz})\end{array}$ & $\begin{array}{c}\text { Obs-Calc } \\
(\mathrm{kHz})\end{array}$ \\
\hline 5 & 1 & 4 & 6 & 4 & 1 & 3 & 5 & 10631.076 & -0.001 \\
5 & 2 & 3 & 6 & 4 & 2 & 2 & 5 & 10716.129 & -0.005 \\
5 & 2 & 3 & 5 & 4 & 2 & 2 & 4 & 10716.138 & 0.000 \\
6 & 0 & 6 & 7 & 5 & 1 & 5 & 6 & 11642.957 & -0.009 \\
6 & 1 & 6 & 7 & 5 & 1 & 5 & 6 & 11714.382 & -0.008 \\
6 & 0 & 6 & 6 & 5 & 0 & 5 & 5 & 11780.726 & 0.001 \\
6 & 0 & 6 & 7 & 5 & 0 & 5 & 6 & 11780.742 & 0.000 \\
\hline
\end{tabular}

Table S5. Observed transitions for $\mathrm{CF}_{3} \mathrm{SO}_{3} \mathrm{D} \cdots \mathrm{DOH}$

\begin{tabular}{cccccccc}
\hline$J^{\prime}$ & $K_{a}{ }^{\prime}$ & $K_{c}{ }^{\prime}$ & $J^{\prime \prime}$ & $K_{a}{ }^{\prime}$ & $K_{c}{ }^{\prime}$ & $\begin{array}{c}\text { Observed Frequency } \\
(\mathrm{MHz})\end{array}$ & $\begin{array}{c}\text { Obs-Calc } \\
(\mathrm{kHz})\end{array}$ \\
\hline 4 & 0 & 4 & 3 & 1 & 3 & 7562.990 & 0.001 \\
4 & 1 & 4 & 3 & 1 & 3 & 7814.839 & -0.001 \\
4 & 0 & 4 & 3 & 0 & 3 & 7967.429 & 0.000 \\
4 & 1 & 4 & 3 & 0 & 3 & 8219.279 & 0.000 \\
4 & 1 & 3 & 3 & 1 & 2 & 8498.993 & 0.000 \\
5 & 0 & 5 & 4 & 1 & 4 & 9590.348 & -0.003 \\
5 & 1 & 5 & 4 & 1 & 4 & 9732.186 & 0.001 \\
5 & 0 & 5 & 4 & 0 & 4 & 9842.202 & 0.000 \\
5 & 1 & 5 & 4 & 0 & 4 & 9984.036 & 0.001 \\
4 & 2 & 3 & 3 & 1 & 2 & 10116.307 & -0.001 \\
3 & 3 & 1 & 2 & 2 & 0 & 10200.027 & -0.002 \\
5 & 2 & 4 & 4 & 2 & 3 & 10205.185 & -0.001 \\
3 & 3 & 0 & 2 & 2 & 1 & 10231.111 & 0.003 \\
5 & 3 & 3 & 4 & 3 & 2 & 10338.335 & 0.000 \\
5 & 3 & 2 & 4 & 3 & 1 & 10395.485 & 0.002 \\
5 & 1 & 4 & 4 & 1 & 3 & 10551.354 & -0.003 \\
5 & 2 & 3 & 4 & 2 & 2 & 10628.655 & -0.005 \\
6 & 0 & 6 & 5 & 1 & 5 & 11561.492 & -0.002 \\
6 & 1 & 6 & 5 & 1 & 5 & 11635.690 & -0.004 \\
6 & 0 & 6 & 5 & 0 & 5 & 11703.317 & -0.010 \\
6 & 1 & 6 & 5 & 0 & 5 & 11777.516 & -0.012 \\
5 & 2 & 4 & 4 & 1 & 3 & 11822.503 & 0.002 \\
4 & 3 & 2 & 3 & 2 & 1 & 12194.137 & 0.001 \\
6 & 2 & 5 & 5 & 2 & 4 & 12196.483 & 0.000 \\
4 & 3 & 1 & 3 & 2 & 2 & 12350.453 & -0.006 \\
6 & 1 & 5 & 5 & 1 & 4 & 12545.485 & -0.001 \\
6 & 2 & 4 & 5 & 2 & 3 & 12805.638 & 0.000 \\
7 & 0 & 7 & 6 & 0 & 6 & 13566.226 & 0.001 \\
\hline & & & & & & & \\
5
\end{tabular}


Table S6. Observed transitions for $\mathrm{CF}_{3} \mathrm{SO}_{3} \mathrm{H} \cdots\left(\mathrm{H}_{2} \mathrm{O}\right)_{2}$

\begin{tabular}{cccccccc}
\hline$J^{\prime}$ & $K_{a}{ }^{\prime}$ & $K_{c}{ }^{\prime}$ & $J^{\prime \prime}$ & $K_{a}{ }^{\prime}$ & $K_{c}{ }^{\prime}$ & $\begin{array}{c}\text { Observed Frequency } \\
(\mathrm{MHz})\end{array}$ & $\begin{array}{c}\text { Obs-Calc } \\
(\mathrm{kHz})\end{array}$ \\
\hline 3 & 2 & 2 & 2 & 2 & 1 & 4267.082 & 0.006 \\
4 & 2 & 3 & 3 & 2 & 2 & 5688.184 & 0.002 \\
4 & 1 & 4 & 3 & 0 & 3 & 6251.941 & -0.004 \\
5 & 1 & 5 & 4 & 1 & 4 & 7006.476 & 0.001 \\
5 & 0 & 5 & 4 & 0 & 4 & 7081.377 & 0.001 \\
5 & 2 & 4 & 4 & 2 & 3 & 7108.215 & -0.001 \\
5 & 2 & 3 & 4 & 2 & 2 & 7138.472 & -0.001 \\
5 & 1 & 4 & 4 & 1 & 3 & 7203.236 & -0.003 \\
5 & 1 & 5 & 4 & 0 & 4 & 7584.387 & -0.002 \\
4 & 2 & 3 & 3 & 1 & 2 & 7839.679 & -0.007 \\
4 & 2 & 2 & 3 & 1 & 3 & 8099.829 & 0.005 \\
6 & 1 & 6 & 5 & 1 & 5 & 8403.976 & 0.000 \\
6 & 0 & 6 & 5 & 0 & 5 & 8482.130 & -0.004 \\
6 & 2 & 5 & 5 & 2 & 4 & 8526.915 & 0.000 \\
6 & 2 & 4 & 5 & 2 & 3 & 8578.611 & -0.001 \\
6 & 1 & 5 & 5 & 1 & 4 & 8638.912 & 0.001 \\
6 & 1 & 6 & 5 & 0 & 5 & 8906.991 & 0.002 \\
5 & 2 & 4 & 4 & 1 & 3 & 9182.711 & -0.002 \\
7 & 0 & 7 & 6 & 1 & 6 & 9451.352 & 0.001 \\
7 & 1 & 7 & 6 & 1 & 6 & 9799.717 & -0.002 \\
7 & 0 & 7 & 6 & 0 & 6 & 9876.202 & -0.004 \\
7 & 2 & 6 & 6 & 2 & 5 & 9944.023 & 0.000 \\
7 & 3 & 5 & 6 & 3 & 4 & 9966.656 & -0.005 \\
7 & 3 & 4 & 6 & 3 & 3 & 9971.380 & 0.002 \\
7 & 2 & 5 & 6 & 2 & 4 & 10023.744 & -0.001 \\
7 & 1 & 6 & 6 & 1 & 5 & 10071.521 & 0.000 \\
7 & 1 & 7 & 6 & 0 & 6 & 10224.574 & 0.000 \\
6 & 2 & 5 & 5 & 1 & 4 & 10506.392 & 0.003 \\
8 & 0 & 8 & 7 & 1 & 7 & 10915.915 & 0.002 \\
8 & 1 & 8 & 7 & 1 & 7 & 11193.639 & -0.004 \\
8 & 0 & 8 & 7 & 0 & 7 & 11264.281 & -0.001 \\
8 & 2 & 7 & 7 & 2 & 6 & 11359.296 & 0.000 \\
8 & 3 & 6 & 7 & 3 & 5 & 11392.252 & 0.002 \\
8 & 3 & 5 & 7 & 3 & 4 & 11401.585 & -0.004 \\
8 & 2 & 6 & 7 & 2 & 5 & 11472.870 & -0.003 \\
8 & 1 & 7 & 7 & 1 & 6 & 11500.280 & 0.000 \\
9 & 1 & 8 & 7 & 0 & 7 & 11542.006 & -0.005 \\
9 & 9 & 8 & 1 & 8 & 12585.765 & 0.000 \\
& & 8 & 8 & 2 & 7 & 12772.518 & 0.012 \\
\hline
\end{tabular}


Table S6. Observed transitions for $\mathrm{CF}_{3} \mathrm{SO}_{3} \mathrm{H} \cdots\left(\mathrm{H}_{2} \mathrm{O}\right)_{2}$ (continued)

\begin{tabular}{cccccccc}
\hline$J^{\prime}$ & $K_{a}{ }^{\prime}$ & $K_{c}{ }^{\prime}$ & $J^{\prime \prime}$ & $K_{a}{ }^{\prime}$ & $K_{c}{ }^{\prime}$ & $\begin{array}{c}\text { Observed Frequency } \\
(\mathrm{MHz})\end{array}$ & $\begin{array}{c}\text { Obs-Calc } \\
(\mathrm{kHz})\end{array}$ \\
\hline 9 & 3 & 7 & 8 & 3 & 6 & 12817.927 & -0.013 \\
9 & 1 & 8 & 8 & 1 & 7 & 12924.308 & 0.000 \\
9 & 2 & 7 & 8 & 2 & 6 & 12924.337 & 0.001 \\
10 & 0 & 10 & 9 & 0 & 9 & 14028.028 & -0.004 \\
10 & 2 & 9 & 9 & 2 & 8 & 14183.452 & 0.001 \\
10 & 1 & 9 & 9 & 1 & 8 & 14342.668 & -0.011 \\
11 & 0 & 11 & 10 & 0 & 10 & 15406.777 & 0.003 \\
11 & 2 & 10 & 10 & 2 & 9 & 15591.962 & -0.002 \\
11 & 1 & 10 & 10 & 1 & 9 & 15754.509 & 0.000 \\
11 & 2 & 9 & 10 & 2 & 8 & 15826.478 & -0.010 \\
\hline
\end{tabular}

Table S7. Observed transitions for $\mathrm{CF}_{3}{ }^{34} \mathrm{SO}_{3} \mathrm{H} \cdots\left(\mathrm{H}_{2} \mathrm{O}\right)_{2}$

\begin{tabular}{cccccccc}
\hline$J^{\prime}$ & $K_{a}{ }^{\prime}$ & $K_{c}{ }^{\prime}$ & $J^{\prime}$ & $K_{a}{ }^{\prime}$ & $K_{c}{ }^{\prime}$ & $\begin{array}{c}\text { Observed Frequency } \\
(\mathrm{MHz})\end{array}$ & $\begin{array}{c}\text { Obs-Calc } \\
(\mathrm{kHz})\end{array}$ \\
\hline 5 & 1 & 5 & 4 & 1 & 4 & 6994.519 & 0.003 \\
5 & 0 & 5 & 4 & 0 & 4 & 7071.065 & 0.002 \\
5 & 1 & 4 & 4 & 1 & 3 & 7198.218 & 0.000 \\
6 & 1 & 6 & 5 & 1 & 5 & 8389.354 & -0.001 \\
6 & 0 & 6 & 5 & 0 & 5 & 8468.658 & -0.001 \\
6 & 2 & 5 & 5 & 2 & 4 & 8516.822 & 0.002 \\
6 & 2 & 4 & 5 & 2 & 3 & 8572.456 & 0.000 \\
6 & 1 & 5 & 5 & 1 & 4 & 8632.464 & -0.002 \\
6 & 1 & 6 & 5 & 0 & 5 & 8879.642 & 0.000 \\
7 & 0 & 7 & 6 & 1 & 6 & 9448.252 & 0.000 \\
7 & 1 & 7 & 6 & 1 & 6 & 9782.325 & 0.001 \\
7 & 0 & 7 & 6 & 0 & 6 & 9859.236 & 0.000 \\
7 & 1 & 6 & 6 & 1 & 5 & 10063.383 & 0.004 \\
8 & 0 & 8 & 7 & 0 & 7 & 11243.653 & 0.001 \\
\hline
\end{tabular}


Table S8. Observed transitions for $\mathrm{CF}_{3} \mathrm{SO}_{3} \mathrm{H} \cdots\left(\mathrm{D}_{2} \mathrm{O}\right)_{2}$

\begin{tabular}{cccccccc}
\hline$J$ & $K_{a}{ }^{\prime}$ & $K_{c}{ }^{\prime}$ & $J^{\prime}$ & $K_{a}{ }^{\prime}$ & $K_{c}{ }^{\prime}$ & $\begin{array}{c}\text { Observed Frequency } \\
(\mathrm{MHz})\end{array}$ & $\begin{array}{c}\text { Obs-Calc } \\
(\mathrm{kHz})\end{array}$ \\
\hline 5 & 1 & 5 & 4 & 1 & 4 & 6634.917 & -0.002 \\
5 & 0 & 5 & 4 & 0 & 4 & 6701.195 & -0.001 \\
5 & 1 & 4 & 4 & 1 & 3 & 6801.408 & -0.001 \\
6 & 1 & 6 & 5 & 1 & 5 & 7959.095 & -0.005 \\
6 & 0 & 6 & 5 & 0 & 5 & 8029.987 & -0.001 \\
6 & 1 & 5 & 5 & 1 & 4 & 8158.172 & 0.000 \\
7 & 1 & 7 & 6 & 1 & 6 & 9281.950 & 0.005 \\
7 & 0 & 7 & 6 & 0 & 6 & 9353.516 & -0.002 \\
7 & 2 & 6 & 6 & 2 & 5 & 9403.380 & -0.001 \\
7 & 2 & 5 & 6 & 2 & 4 & 9461.793 & -0.001 \\
7 & 1 & 6 & 6 & 1 & 5 & 9512.807 & 0.006 \\
8 & 1 & 8 & 7 & 1 & 7 & 10603.372 & -0.005 \\
8 & 0 & 8 & 7 & 0 & 7 & 10671.984 & -0.005 \\
8 & 2 & 7 & 7 & 2 & 6 & 10742.871 & 0.004 \\
8 & 2 & 6 & 7 & 2 & 5 & 10827.110 & -0.006 \\
9 & 0 & 9 & 8 & 0 & 8 & 11986.100 & -0.006 \\
\hline
\end{tabular}


Table S9. Observed transitions for $\mathrm{CF}_{3} \mathrm{SO}_{3} \mathrm{H} \cdots\left(\mathrm{H}_{2} \mathrm{O}\right)_{3}$

\begin{tabular}{cccccccc}
\hline$J$ & $K_{a}{ }^{\prime}$ & $K_{c}{ }^{\prime}$ & $J^{\prime \prime}$ & $K_{a}{ }^{\prime}$ & $K_{c}{ }^{\prime}$ & $\begin{array}{c}\text { Observed Frequency } \\
(\mathrm{MHz})\end{array}$ & $\begin{array}{c}\text { Obs-Calc } \\
(\mathrm{kHz})\end{array}$ \\
\hline 6 & 0 & 6 & 5 & 0 & 5 & 6054.493 & 0.002 \\
6 & 2 & 5 & 5 & 2 & 4 & 6157.366 & -0.003 \\
6 & 1 & 5 & 5 & 1 & 4 & 6335.036 & 0.006 \\
7 & 1 & 7 & 6 & 1 & 6 & 6934.579 & -0.003 \\
7 & 0 & 7 & 6 & 0 & 6 & 7024.347 & 0.008 \\
7 & 2 & 5 & 6 & 2 & 4 & 7352.673 & -0.005 \\
7 & 1 & 6 & 6 & 1 & 5 & 7372.402 & 0.006 \\
8 & 1 & 8 & 7 & 1 & 7 & 7912.796 & 0.001 \\
8 & 0 & 8 & 7 & 0 & 7 & 7986.595 & 0.003 \\
8 & 2 & 7 & 7 & 2 & 6 & 8186.828 & -0.003 \\
8 & 3 & 6 & 7 & 3 & 5 & 8261.998 & 0.010 \\
8 & 3 & 5 & 7 & 3 & 4 & 8294.689 & -0.012 \\
8 & 1 & 7 & 7 & 1 & 6 & 8399.538 & 0.002 \\
8 & 2 & 6 & 7 & 2 & 5 & 8431.245 & -0.001 \\
9 & 1 & 9 & 8 & 1 & 8 & 8887.894 & -0.004 \\
9 & 0 & 9 & 8 & 0 & 8 & 8945.019 & 0.002 \\
7 & 1 & 6 & 6 & 0 & 6 & 9077.982 & 0.000 \\
9 & 2 & 8 & 8 & 2 & 7 & 9194.840 & -0.012 \\
9 & 3 & 6 & 8 & 3 & 5 & 9354.655 & -0.012 \\
9 & 1 & 8 & 8 & 1 & 7 & 9414.248 & -0.004 \\
9 & 2 & 7 & 8 & 2 & 6 & 9508.031 & 0.005 \\
10 & 1 & 10 & 9 & 1 & 9 & 9860.312 & 0.000 \\
10 & 0 & 10 & 9 & 0 & 9 & 9902.442 & 0.001 \\
10 & 2 & 9 & 9 & 2 & 8 & 10197.948 & 0.003 \\
10 & 3 & 8 & 9 & 3 & 7 & 10329.851 & 0.000 \\
10 & 1 & 9 & 9 & 1 & 8 & 10414.796 & 0.004 \\
10 & 3 & 7 & 9 & 3 & 6 & 10424.488 & 0.004 \\
8 & 1 & 7 & 7 & 0 & 7 & 10453.185 & 0.005 \\
10 & 2 & 8 & 9 & 2 & 7 & 10579.099 & 0.002 \\
11 & 1 & 11 & 10 & 1 & 10 & 10830.512 & 0.004 \\
11 & 0 & 11 & 10 & 0 & 10 & 10860.432 & -0.005 \\
11 & 2 & 10 & 10 & 2 & 9 & 11195.943 & -0.003 \\
11 & 3 & 8 & 10 & 3 & 7 & 11504.239 & -0.007 \\
12 & 12 & 11 & 1 & 11 & 11798.958 & 0.000 \\
11 & 0 & 11 & 11819.602 & -0.007 \\
\hline
\end{tabular}


Table S10. Comparison between Observed Rotational Constants for Parent Triflic Acid Monohydrate and Theoretical Values for Predicted Conformers. ${ }^{a, b}$

\begin{tabular}{|c|c|c|c|c|c|c|}
\hline & $\begin{array}{c}\text { M06-2X } \\
6-311++\mathrm{G}(3 \mathrm{df}, 3 \mathrm{pd})\end{array}$ & $\begin{array}{c}\text { B3LYP-D3BJ } \\
\text { def2-TZVP }\end{array}$ & $\begin{array}{l}\text { B3LYP-D3BJ } \\
\text { jun-cc-pVDZc }\end{array}$ & $\begin{array}{l}\text { B2PLYP-D3BJ } \\
\text { jun-cc-pVTZc }\end{array}$ & $\begin{array}{c}\text { DSD-PBEP86-D3BJ } \\
\text { jun-cc-pVTZ }\end{array}$ & $\mathrm{RMS}^{\mathrm{d}}{ }^{\mathrm{d}}$ \\
\hline$A$ (observed) & $1853.10915(22)$ & & & & & \\
\hline \multirow[t]{2}{*}{ IA } & 1878 & 1866 & 1834 & 1866 & 1866 & 0.9 \\
\hline & 1877 & 1865 & 1833 & 1864 & 1864 & 0.9 \\
\hline \multirow[t]{2}{*}{ IB } & 1838 & 1819 & 1806 & 1838 & 1837 & 1.5 \\
\hline & 1829 & 1811 & 1797 & 1829 & 1828 & 2.0 \\
\hline$B$ (observed) & $1144.85988(10)$ & & & & & \\
\hline \multirow[t]{2}{*}{ IA } & 1167 & 1117 & 1107 & 1123 & 1139 & 2.2 \\
\hline & 1148 & 1097 & 1088 & 1104 & 1120 & 3.5 \\
\hline \multirow[t]{2}{*}{ IB } & 1199 & 1151 & 1126 & 1141 & 1159 & 2.3 \\
\hline & 1184 & 1136 & 1112 & 1127 & 1144 & 2.2 \\
\hline$C$ (observed) & $959.540437(79)$ & & & & & \\
\hline \multirow[t]{2}{*}{ IA } & 980 & 947 & 938 & 951 & 961 & 1.6 \\
\hline & 967 & 934 & 924 & 938 & 948 & 2.4 \\
\hline \multirow[t]{2}{*}{ IB } & 991 & 958 & 944 & 957 & 967 & 1.7 \\
\hline & 979 & 947 & 932 & 945 & 956 & 1.8 \\
\hline$\left|\mu_{a}\right|$ & $\neq \mathbf{0}^{\mathrm{e}}$ & & & & & \\
\hline$\left|\mu_{b}\right|$ & $\neq 0^{\mathrm{e}}$ & & & & & \\
\hline \multirow[t]{2}{*}{$\left|\mu_{c}\right|$} & $<<\boldsymbol{\mu}_{a}, \boldsymbol{\mu}_{b}{ }^{\mathrm{e}}$ & & & & & \\
\hline & $\begin{array}{c}\text { M06-2X } \\
6-311++G(3 d f .3 p d)\end{array}$ & $\begin{array}{l}\text { B3LYP-D3BJ } \\
\text { def2-TZVP }\end{array}$ & $\begin{array}{l}\text { B3LYP-D3BJ } \\
\text { jun-cc-pVDZc }\end{array}$ & $\begin{array}{l}\text { B2PLYP-D3BJ } \\
\text { iun-cc-pVTZc }\end{array}$ & $\begin{array}{l}\text { DSD-PBEP86-D3BJ } \\
\text { jun-cc-pVTZc }\end{array}$ & \\
\hline$\left(\mu_{a}, \mu_{b}, \mu_{c}\right.$ & $\left(3.8,2.1,0.0^{\mathrm{f}}\right)$ & $(3.8,2,0,0.1)$ & $(3.9,2.1,0.2)$ & $\left(3.7,1.9,0.0^{f}\right)$ & $\left(3.7,2.0,0.0^{\mathrm{f}}\right)$ & \\
\hline$\left(\mu_{a}, \mu_{b}, \mu_{c}\right.$ & $(2.1,3.9,0.1)$ & $(2.1,3.9,0.3)$ & $(2.3,4.0,0.3)$ & $(2.2,3.8,0.2)$ & $(2.1,3.8,0.2)$ & \\
\hline
\end{tabular}

(a) Fitted rotational constants of water tunneling state $0^{-}$and qualitative observations about the dipole moment components are in bold. See Table 1 in the text for the complete set of fitted spectroscopic constants for triflic acid monohydrate. Rotational constants are in MHz. Dipole moment components are in Debye.

(b) Equilibrium rotational constants calculated for each level of theory are presented in the first row for $A, B$, and $C$. Vibrational ground state rotational constants were calculated using the vibrational corrections at the B3LYP-D3BJ/jun-cc-pVDZ level/basis and are given in the second row for $A, B$, and $C$.

(c) For calculations using the jun-cc-pVDZ or jun-cc-pVTZ basis sets, the jun-cc-pV(D+d)Z or jun-cc-pV(T+d)Z basis set was used on the sulfur atom, respectively.

(d) RMS percent deviation of calculated rotational constants relative to observed values among the five levels of theory used.

(e) From the observation of $a$ - and $b$-type transitions. No $c$-type transitions were observed.

(f) The dipole moment component along the principal $c$-axis is not identically zero. 
Table S11. Observed and Calculated Isotope Shifts for Triflic Acid Monohydrate Isotopologues. ${ }^{a}$

\begin{tabular}{|c|c|c|c|c|c|c|}
\hline \multicolumn{7}{|c|}{$\mathrm{CF}_{3}{ }^{34} \mathrm{SO}_{3} \mathrm{H} \cdots \mathrm{H}_{2} \mathrm{O}$} \\
\hline \multicolumn{7}{|c|}{$\Delta A[\mathrm{MHz}]=-8.45039(44)$} \\
\hline \multicolumn{7}{|c|}{$\Delta B[\mathrm{MHz}]=\mathbf{- 0 . 2 9 2 2 1 ( 1 9 )}$} \\
\hline \multicolumn{7}{|c|}{$\Delta C[\mathrm{MHz}]=\mathbf{- 2 . 4 2 8 5 2 ( 1 5 )}$} \\
\hline & & M06-2X & B3LYP-D3BJ & B3LYP-D3BJ & B2PLYP-D3BJ & DSD-PBEP86-D3BJ \\
\hline & & $6-311++\mathrm{G}(3 \mathrm{df}, 3 \mathrm{pd})$ & def2-TZVP & jun-cc-pVDZ & jun-cc-pVTZ & jun-cc-pVTZ \\
\hline \multirow[t]{3}{*}{ IA } & $\Delta A$ & -8.5 & -8.2 & -8.1 & -8.3 & -8.4 \\
\hline & $\Delta B$ & -0.4 & -0.4 & -0.4 & -0.4 & -0.4 \\
\hline & $\Delta C$ & -2.5 & -2.4 & -2.4 & -2.4 & -2.4 \\
\hline \multirow[t]{3}{*}{ IB } & $\Delta A$ & -8.6 & -8.4 & -8.2 & -8.3 & -8.4 \\
\hline & $\Delta B$ & -0.4 & -0.4 & -0.4 & -0.4 & -0.4 \\
\hline & $\Delta C$ & -2.7 & -2.6 & -2.5 & -2.5 & -2.6 \\
\hline \multicolumn{7}{|c|}{$\mathrm{CF}_{3} \mathrm{SO}_{3} \mathrm{H} \cdots \mathrm{D}_{2} \mathrm{O}$} \\
\hline \multicolumn{7}{|c|}{$\Delta A[\mathrm{MHz}]=\mathbf{- 3 3 . 5 8 1 6 ( 2 7 )}$} \\
\hline \multicolumn{7}{|c|}{$\Delta B[\mathrm{MHz}]=\mathbf{- 4 2 . 2 9 7 9 7 ( 3 7 )}$} \\
\hline \multicolumn{7}{|c|}{$\Delta C[\mathrm{MHz}]=\mathbf{- 3 6 . 1 0 1 8 6 ( 3 4 )}$} \\
\hline & & M06-2X & B3LYP-D3BJ & B3LYP-D3BJ & B2PLYP-D3BJ & DSD-PBEP86-D3BJ \\
\hline & & $6-311++\mathrm{G}(3 \mathrm{df}, 3 \mathrm{pd})$ & def2-TZVP & jun-cc-pVDZ & jun-cc-pVTZ & jun-cc-pVTZ \\
\hline \multirow[t]{3}{*}{ IA } & $\Delta A$ & -14.5 & -14.4 & -15.1 & -14.1 & -14.0 \\
\hline & $\Delta B$ & -57.9 & -55.3 & -55.6 & -56.3 & -57.2 \\
\hline & $\Delta C$ & -42.2 & -41.1 & -41.4 & -41.6 & -41.9 \\
\hline \multirow[t]{3}{*}{ IB } & $\Delta A$ & -32.7 & -32.7 & -32.4 & -32.6 & -32.5 \\
\hline & $\Delta B$ & -44.0 & -43.2 & -44.0 & -43.9 & -44.1 \\
\hline & $\Delta C$ & -36.7 & -36.3 & -37.0 & -36.9 & -37.0 \\
\hline \multicolumn{7}{|c|}{$\mathrm{CF}_{3} \mathrm{SO}_{3} \mathrm{H} \cdots \mathrm{DOH}$} \\
\hline \multicolumn{7}{|c|}{$\Delta A[\mathrm{MHz}]=-7.5224(32)$} \\
\hline \multicolumn{7}{|c|}{$\Delta B[\mathrm{MHz}]=-\mathbf{2 1 . 0 2 3 9 8 ( 7 4 )}$} \\
\hline \multicolumn{7}{|c|}{$\Delta C[\mathrm{MHz}]=-\mathbf{1 4 . 2 5 6 2 2 ( 3 7 )}$} \\
\hline & & M06-2X & B3LYP-D3BJ & B3LYP-D3BJ & B2PLYP-D3BJ & DSD-PBEP86-D3BJ \\
\hline & & $6-311++\mathrm{G}(3 \mathrm{df}, 3 \mathrm{pd})$ & def2-TZVP & jun-cc-pVDZ & jun-cc-pVTZ & jun-cc-pVTZ \\
\hline \multirow[t]{3}{*}{ IA } & $\Delta A$ & -9.1 & -9.4 & -9.3 & -9.0 & -9.0 \\
\hline & $\Delta B$ & -20.7 & -19.9 & -20.4 & -20.5 & -20.8 \\
\hline & $\Delta C$ & -14.5 & -14.2 & -14.6 & -14.6 & -14.6 \\
\hline \multirow[t]{3}{*}{ IB } & $\Delta A$ & -8.7 & -8.6 & -9.1 & -8.8 & -8.8 \\
\hline & $\Delta B$ & -21.5 & -21.0 & -20.9 & -21.0 & -21.2 \\
\hline & $\Delta C$ & -14.6 & -14.5 & -14.6 & -14.6 & -14.7 \\
\hline \multicolumn{7}{|c|}{$\mathrm{CF}_{3} \mathrm{SO}_{3} \mathrm{D} \cdots \mathrm{DOH}$} \\
\hline \multicolumn{7}{|c|}{$\Delta A[\mathrm{MHz}]=-\mathbf{1 6 . 0 7 8 1 ( 1 5 )}$} \\
\hline \multicolumn{7}{|c|}{$\Delta B[\mathrm{MHz}]=-\mathbf{3 0 . 4 1 6 1 2 ( 5 3 )}$} \\
\hline \multicolumn{7}{|c|}{$\Delta C[\mathrm{MHz}]=-\mathbf{2 0 . 4 0 4 2 0}(\mathbf{2 6})$} \\
\hline & & M06-2X & B3LYP-D3BJ & B3LYP-D3BJ & B2PLYP-D3BJ & DSD-PBEP86-D3BJ \\
\hline & & $6-311++\mathrm{G}(3 \mathrm{df}, 3 \mathrm{pd})$ & def2-TZVP & jun-cc-pVDZ & jun-cc-pVTZ & jun-cc-pVTZ \\
\hline IA & $\Delta A$ & -14.7 & -15.0 & -14.7 & -14.5 & -14.4 \\
\hline & $\Delta B$ & -31.4 & -29.9 & -30.3 & -30.6 & -31.0 \\
\hline & $\Delta C$ & -20.7 & -20.3 & -20.5 & -20.6 & -20.7 \\
\hline IB & $\Delta A$ & -13.9 & -13.8 & -14.2 & -14.1 & -13.9 \\
\hline & $\Delta B$ & -32.7 & -31.5 & -31.1 & -31.2 & -31.7 \\
\hline & $\Delta C$ & -20.9 & -20.5 & -20.5 & -20.6 & -20.7 \\
\hline
\end{tabular}

(a) Predicted isotope shifts are calculated from theoretical equilibrium constants 
Table S12. Comparison of Observed Rotational Constants for Parent Triflic Acid Dihydrate and Theoretical Values for Predicted Conformers.a,b,c

\begin{tabular}{|c|c|c|c|c|c|c|}
\hline & $\begin{array}{c}\text { M06-2X } \\
6-311++G(3 d f, 3 p d)\end{array}$ & $\begin{array}{c}\text { B3LYP-D3BJ } \\
\text { def2-TZVP }\end{array}$ & $\begin{array}{l}\text { B3LYP-D3BJ } \\
\text { jun-cc-pVDZ }\end{array}$ & $\begin{array}{l}\text { B2PLYP-D3BJ } \\
\text { jun-cc-pVTZ }\end{array}$ & $\begin{array}{c}\text { DSD-PBEP86-D3BJ } \\
\text { jun-cc-pVTZ }\end{array}$ & $\mathrm{RMS} \% \mathrm{e}^{\mathrm{e}}$ \\
\hline$A$ (observed) & $1467.5486(28)$ & & & & & \\
\hline \multirow[t]{2}{*}{ IIA } & 1472 & 1424 & 1397 & 1444 & 1451 & 2.7 \\
\hline & 1476 & 1427 & 1401 & 1447 & 1455 & 2.5 \\
\hline \multirow[t]{2}{*}{ IIB } & 1376 & 1372 & 1369 & 1400 & 1393 & 5.9 \\
\hline & 1374 & 1370 & 1368 & 1398 & 1391 & 6.0 \\
\hline \multirow[t]{2}{*}{ IIC } & 1499 & 1446 & 1428 & 1465 & 1477 & 1.7 \\
\hline & 1507 & 1453 & 1436 & 1473 & 1485 & 1.7 \\
\hline \multirow[t]{2}{*}{ IID } & 1385 & 1400 & 1382 & 1419 & 1406 & 4.8 \\
\hline & 1390 & 1406 & 1387 & 1425 & 1412 & 4.4 \\
\hline$B$ (observed) & $730.94197(26)$ & & & & & \\
\hline \multirow[t]{2}{*}{ IIA } & 761 & 752 & 748 & 735 & 741 & 2.5 \\
\hline & 754 & 746 & 742 & 729 & 735 & 1.8 \\
\hline \multirow[t]{2}{*}{ IIB } & 859 & 801 & 771 & 764 & 784 & 10.0 \\
\hline & 856 & 799 & 769 & 762 & 782 & 9.7 \\
\hline \multirow[t]{2}{*}{ IIC } & 758 & 744 & 733 & 730 & 735 & 1.9 \\
\hline & 752 & 737 & 727 & 723 & 729 & 1.4 \\
\hline \multirow{2}{*}{ IID } & 835 & 760 & 753 & 742 & 765 & 7.1 \\
\hline & 823 & 748 & 741 & 730 & 753 & 5.9 \\
\hline$C$ (observed) & $691.42664(21)$ & & & & & \\
\hline \multirow[t]{2}{*}{ IIA } & 710 & 703 & 700 & 693 & 698 & 1.6 \\
\hline & 704 & 698 & 694 & 688 & 692 & 1.0 \\
\hline \multirow[t]{2}{*}{ IIB } & 756 & 724 & 709 & 709 & 721 & 5.3 \\
\hline & 751 & 719 & 704 & 704 & 716 & 4.7 \\
\hline \multirow[t]{2}{*}{ IIC } & 699 & 693 & 685 & 684 & 686 & 0.9 \\
\hline & 692 & 686 & 678 & 677 & 679 & 1.6 \\
\hline \multirow[t]{2}{*}{ IID } & 747 & 708 & 702 & 700 & 713 & 4.1 \\
\hline & 738 & 699 & 693 & 691 & 704 & 3.1 \\
\hline$\left|\mu_{a}\right|$ & $\neq 0^{\mathrm{f}}$ & & & & & \\
\hline$\left|\mu_{b}\right|$ & $\neq \mathbf{0}^{\mathrm{f}}$ & & & & & \\
\hline \multirow[t]{2}{*}{$\left|\mu_{c}\right|$} & $<<\mu_{a}, \mu_{b}{ }^{\mathrm{f}}$ & & & & & \\
\hline & $\begin{array}{c}\text { M06-2X } \\
6-311++\mathrm{G}(3 \mathrm{df}, 3 \mathrm{pd})\end{array}$ & $\begin{array}{l}\text { B3LYP-D3BJ } \\
\text { def2-TZVP }\end{array}$ & $\begin{array}{l}\text { B3LYP-D3BJ } \\
\text { jun-cc-pVDZ }\end{array}$ & $\begin{array}{l}\text { B2PLYP-D3BJ } \\
\text { jun-cc-pVTZ }\end{array}$ & $\begin{array}{l}\text { DSD-PBEP86-D3BJ } \\
\text { jun-cc-pVTZ }\end{array}$ & \\
\hline IIA $\left(\mu_{a}, \mu_{b}, \mu_{c}\right.$ & $(3.6,2.3,0.1)$ & $\left(3.6,2.3,0.0^{\mathrm{g}}\right)$ & $\left(3.7,2.4,0.0^{\mathrm{g}}\right)$ & $(3.5,2.3,0.1)$ & $(3.4,2.2,0.1)$ & \\
\hline IIB $\quad\left(\mu_{a}, \mu_{b}, \mu_{c}\right.$ & $(3.7,2.0,0.9)$ & $(3.6,2.1,0.8)$ & $(3.8,2.3,0.6)$ & $(3.6,2.1,0.8)$ & $(3.6,2.1,0.8)$ & \\
\hline IIC $\quad\left(\mu_{a}, \mu_{b}, \mu_{c}\right.$ & $(2.9,3.8,1.8)$ & $(2.7,4.1,1.5)$ & $(2.9,4.0,1.5)$ & $(2.7,3.8,1.5)$ & $(2.7,3.8,1.7)$ & \\
\hline IID $\quad\left(\mu_{a}, \mu_{b}, \mu_{c}\right.$ & $(5.1,0.6,0.1)$ & $(5.0,0.3,0.3)$ & $(4.9,0.5,0.2)$ & $(4.7,0.4,0.5)$ & $(4.8,0.3,0.3)$ & \\
\hline
\end{tabular}

(a) Fitted rotational constants and qualitative observations about the dipole moment components are in bold.

See Table 2 in the text for the complete set of fitted spectroscopic constants for triflic acid dihydrate.

Rotational constants are in MHz. Dipole moment components are in Debye.

(b) Equilibrium rotational constants calculated for each level of theory are presented in the first row for $A, B$, and $C$. Vibrational ground state rotational constants were calculated using the vibrational corrections at the B3LYP-D3BJ/jun-cc-pVDZ level/basis and are given in the second row for $A, B$, and $C$.

(c) The lowest energy conformer is consistently calculated to be IIA and is highlighted in bold.

(d) For calculations using the jun-cc-pVDZ or jun-cc-pVTZ basis sets, the jun-cc-pV(D+d)Z or jun-cc$\mathrm{pV}(\mathrm{T}+\mathrm{d}) \mathrm{Z}$ basis set was used on the sulfur atom, respectively.

(e) RMS percent deviation of calculated rotational constants relative to observed values among the five levels of theory used.

(f) From the observation of $a$ - and $b$-type transitions. No $c$-type transitions were observed.

(g) The dipole moment component along the principal $c$-axis is not identically zero. 
Table S13. Observed and Calculated Isotope Shifts for Triflic Acid Dihydrate Isotopologues.a,b

\begin{tabular}{|c|c|c|c|c|c|c|}
\hline & & & $\mathrm{CF}$ & ${ }_{3} \mathrm{H}^{\prime} \cdots\left(\mathrm{H}_{2} \mathrm{O}\right)_{2}$ & & \\
\hline$\Delta A[$ & $\mathrm{Hz}]=$ & $446(13)$ & & & & \\
\hline$\Delta B$ & $\mathrm{~Hz}]=$ & $841(20)$ & & & & \\
\hline$\Delta C$ & $\mathrm{~Hz}]=$ & $50274(86)$ & & & & \\
\hline & & M06-2X & B3LYP-D3BJ & B3LYP-D3BJ & B2PLYP-D3BJ & DSD-PBEP86-D3BJ \\
\hline & & $6-311++G(3 d f, 3 p d)$ & def2-TZVP & jun-cc-pVDZ & jun-cc-pVTZ & jun-cc-pVTZ \\
\hline IIA & $\Delta A$ & -6.7 & -6.5 & -6.4 & -6.4 & -6.4 \\
\hline & $\Delta B$ & -0.1 & -0.1 & -0.1 & -0.1 & -0.1 \\
\hline & $\Delta C$ & -1.6 & -1.7 & -1.7 & -1.6 & -1.6 \\
\hline IIB & $\Delta A$ & -6.5 & -6.4 & -6.3 & -6.3 & -6.4 \\
\hline & $\Delta B$ & -0.7 & -0.5 & -0.4 & -0.3 & -0.4 \\
\hline & $\Delta C$ & -2.0 & -1.8 & -1.7 & -1.6 & -1.7 \\
\hline IIC & $\Delta A$ & -6.7 & -6.5 & -6.4 & -6.4 & -6.4 \\
\hline & $\Delta B$ & -0.1 & -0.1 & -0.1 & -0.1 & -0.1 \\
\hline & $\Delta C$ & -1.5 & -1.5 & -1.5 & -1.4 & -1.4 \\
\hline IID & $\Delta A$ & -6.4 & -6.3 & -6.3 & -6.3 & -6.3 \\
\hline & $\Delta B$ & -0.6 & -0.3 & -0.3 & -0.2 & -0.3 \\
\hline & $\Delta C$ & -1.9 & -1.6 & -1.7 & -1.6 & -1.7 \\
\hline & & & & ${ }_{3} \cdots\left(\mathrm{D}_{2} \mathrm{O}\right)_{2}$ & & \\
\hline$\Delta A[$ & $\mathrm{Hz}]=$ & $.472(47)$ & & & & \\
\hline$\Delta B$ & $\mathrm{~Hz}]=$ & $.9218(11)$ & & & & \\
\hline$\Delta C$ & $\mathrm{~Hz}]=$ & $.80506(70)$ & & & & \\
\hline & & M06-2X & B3LYP-D3BJ & B3LYP-D3BJ & B2PLYP-D3BJ & DSD-PBEP86-D3BJ \\
\hline & & $6-311++\mathrm{G}(3 \mathrm{df}, 3 \mathrm{pd})$ & def2-TZVP & jun-cc-pVDZ & jun-cc-pVTZ & jun-cc-pVTZ \\
\hline IIA & $\Delta A$ & -37.9 & -37.6 & -37.5 & -39.0 & -38.7 \\
\hline & $\Delta B$ & -44.1 & -44.6 & -45.6 & -43.5 & -43.7 \\
\hline & $\Delta C$ & -37.7 & -36.2 & -35.4 & -36.6 & -37.0 \\
\hline IIB & $\Delta A$ & -37.1 & -38.3 & -39.2 & -40.6 & -39.8 \\
\hline & $\Delta B$ & -47.0 & -45.2 & -45.3 & -45.0 & -45.5 \\
\hline & $\Delta C$ & -38.2 & -36.9 & -36.3 & -36.6 & -37.2 \\
\hline IIC & $\Delta A$ & -45.2 & -45.3 & -45.0 & -46.7 & -46.6 \\
\hline & $\Delta B$ & -42.2 & -41.7 & -42.0 & -41.2 & -41.4 \\
\hline & $\Delta C$ & -36.0 & -34.5 & -34.6 & -35.0 & -35.4 \\
\hline IID & $\Delta A$ & -34.8 & -35.8 & -36.1 & -37.2 & -36.4 \\
\hline & $\Delta B$ & -54.0 & -50.4 & -50.5 & -49.9 & -51.1 \\
\hline & $\Delta C$ & -40.5 & -37.7 & -37.4 & -37.5 & -38.5 \\
\hline
\end{tabular}

(a) The lowest energy conformer is consistently calculated to be IIA and is highlighted in bold.

(b) Predicted isotope shifts are calculated from theoretical equilibrium constants. 
Table S14. Comparison of Observed Rotational Constants for Parent Triflic Acid Trihydrate and Theoretical Values for Predicted Conformers. ${ }^{\text {a,b,c }}$

\begin{tabular}{|c|c|c|c|c|c|c|c|}
\hline \multirow{2}{*}{\multicolumn{2}{|c|}{$A$ (observed) }} & \multirow{2}{*}{$\begin{array}{c}\text { M06-2X } \\
\text { 6-311++G(3df,3pd) } \\
\mathbf{1 3 5 3 . 4 5 9 9 ( 5 0 )}\end{array}$} & \multirow{2}{*}{$\begin{array}{c}\text { B3LYP-D3BJ } \\
\text { def2-TZVP }\end{array}$} & \multirow[t]{2}{*}{$\begin{array}{l}\text { B3LYP-D3BJ } \\
\text { jun-cc-pVDZ }\end{array}$} & \multirow[t]{2}{*}{$\begin{array}{l}\text { B2PLYP-D3BJ } \\
\text { jun-cc-pVTZ }\end{array}$} & \multirow[t]{2}{*}{$\begin{array}{c}\text { DSD-PBEP86-D3BJ } \\
\text { jun-cc-pVTZ }\end{array}$} & \multirow[t]{2}{*}{$\mathrm{RMS} \% \mathrm{e}$} \\
\hline & & & & & & & \\
\hline \multirow{2}{*}{\multicolumn{2}{|c|}{ IIIA }} & 1376 & 1370 & 1360 & 1366 & 1370 & 1.2 \\
\hline & & 1365 & 1360 & 1350 & 1356 & 1360 & 0.5 \\
\hline \multirow{2}{*}{\multicolumn{2}{|c|}{ IIIB }} & 1383 & 1375 & 1363 & 1366 & 1372 & 1.4 \\
\hline & & 1370 & 1362 & 1350 & 1353 & 1359 & 0.6 \\
\hline \multirow{2}{*}{\multicolumn{2}{|c|}{ IIIC }} & 1028 & 1016 & 1008 & 1019 & 1021 & 24.8 \\
\hline & & 1023 & 1012 & 1003 & 1014 & 1017 & 25.1 \\
\hline \multirow{2}{*}{\multicolumn{2}{|c|}{ IIID }} & 1452 & 1468 & 1451 & 1479 & 1483 & 8.4 \\
\hline & & 1388 & 1404 & 1387 & 1415 & 1420 & 3.8 \\
\hline \multicolumn{3}{|r|}{$546.99304(30)$} & & & & & \\
\hline \multirow{2}{*}{\multicolumn{2}{|c|}{ IIIA }} & 552 & 542 & 536 & 543 & 546 & 1.2 \\
\hline & & 550 & 539 & 533 & 541 & 544 & 1.5 \\
\hline \multirow{2}{*}{\multicolumn{2}{|c|}{ IIIB }} & 552 & 541 & 535 & 543 & 546 & 1.2 \\
\hline & & 550 & 539 & 533 & 541 & 544 & 1.4 \\
\hline \multirow{2}{*}{\multicolumn{2}{|c|}{ IIIC }} & 695 & 631 & 617 & 619 & 636 & 17.7 \\
\hline & & 701 & 637 & 623 & 625 & 642 & 18.8 \\
\hline \multirow{2}{*}{\multicolumn{2}{|c|}{ IIID }} & 556 & 513 & 512 & 512 & 518 & 5.5 \\
\hline & & 540 & 497 & 496 & 497 & 502 & 8.0 \\
\hline \multirow{3}{*}{\multicolumn{2}{|c|}{$\begin{array}{l}C \text { (observed) } \\
\text { IIIA }\end{array}$}} & $482.05570(23)$ & & & & & \\
\hline & & 486 & 480 & 476 & 480 & 483 & 0.7 \\
\hline & & 483 & 477 & 474 & 477 & 480 & 1.0 \\
\hline \multirow{2}{*}{\multicolumn{2}{|c|}{ IIIB }} & 488 & 481 & 478 & 481 & 484 & 0.7 \\
\hline & & 485 & 478 & 475 & 478 & 481 & 0.9 \\
\hline \multirow{2}{*}{\multicolumn{2}{|c|}{ IIIC }} & 601 & 566 & 557 & 558 & 568 & 18.5 \\
\hline & & 603 & 568 & 559 & 560 & 570 & 18.9 \\
\hline \multirow{2}{*}{\multicolumn{2}{|c|}{ IIID }} & 478 & 443 & 442 & 444 & 448 & 7.0 \\
\hline & & 461 & 427 & 426 & 427 & 432 & 10.2 \\
\hline \multirow{4}{*}{\multicolumn{2}{|c|}{$\begin{array}{l}\left|\boldsymbol{\mu}_{\boldsymbol{a}}\right| \\
\left|\boldsymbol{\mu}_{\boldsymbol{b}}\right| \\
\left|\boldsymbol{\mu}_{c}\right|\end{array}$}} & $\pm 0^{\mathrm{f}}$ & & & & & \\
\hline & & $<<\mu_{a}, \mu_{c}{ }^{\mathrm{f}}$ & & & & & \\
\hline & & $\pm 0^{\mathrm{r}}$ & & & & & \\
\hline & & $\begin{array}{c}\text { M06-2X } \\
6-311++G(3 d f, 3 p d) \\
\end{array}$ & $\begin{array}{c}\text { B3LYP-D3BJ } \\
\text { def2-TZVP }\end{array}$ & $\begin{array}{l}\text { B3LYP-D3BJ } \\
\text { jun-cc-pVDZ }\end{array}$ & $\begin{array}{l}\text { B2PLYP-D3BJ } \\
\text { jun-cc-pVTZ }\end{array}$ & $\begin{array}{c}\text { DSD-PBEP86-D3BJ } \\
\text { jun-cc-pVTZ }\end{array}$ & \\
\hline IIIA & $\left(\mu_{a}, \mu_{b}, \mu_{c}\right)$ & $(4.1,0.0,1.3)$ & $(4.1,0.0,1.3)$ & $(4.3,0.0,1.1)$ & $(4.3,0.0,1.3)$ & $(4.3,0.0,1.4)$ & \\
\hline IIIB & $\left(\mu_{a}, \mu_{b}, \mu_{c}\right)$ & $(4.6,1.4,0.5)$ & $(4.6,1.4,0.5)$ & $(4.8,1.3,0.5)$ & $(4.8,1.3,0.4)$ & $(4.8,1.3,0.4)$ & \\
\hline IIIC & $\left(\mu_{a}, \mu_{b}, \mu_{c}\right)$ & $\left(4.6,0.6,0.0^{g}\right)$ & $(4.6,0.5,0.1)$ & $(4.6,0.7,0.1)$ & $(4.4,0.5,0.2)$ & $(4.4,0.5,0.1)$ & \\
\hline IIID & $\left(\mu_{a}, \mu_{b}, \mu_{c}\right)$ & $(3.9,2.0,0.7)$ & $(4.7,1.0,0.5)$ & $(4.8,0.8,0.4)$ & $(4.9,1.0,0.3)$ & $(4.9,1.0,0.3)$ & \\
\hline
\end{tabular}

(a) Fitted rotational constants and qualitative observations about the dipole moment components are in bold. See Table 3 in the text for the complete set of fitted spectroscopic constants for triflic acid trihydrate. Rotational constants are in MHz. Dipole moment components are in Debye.

(b) Equilibrium rotational constants calculated for each level of theory are presented in the first row for $A, B$, and $C$. Vibrational ground state rotational constants were calculated using the vibrational corrections at the B3LYP-D3BJ/jun-cc-pVDZ level/basis and are given in the second row for $A, B$, and $C$.

(c) The lowest energy conformer is consistently calculated to be IIIA and is highlighted in bold.

(d) For calculations using the jun-cc-pVDZ or jun-cc-pVTZ basis sets, the jun-cc-pV(D+d)Z or jun-cc$\mathrm{pV}(\mathrm{T}+\mathrm{d}) \mathrm{Z}$ basis set were used on the sulfur atom, respectively.

(e) RMS percent deviation of calculated rotational constants relative to observed values among the five levels of theory used.

(f) From the observation of $a$ - and $c$-type transitions. No $b$-type transitions were observed.

(g) The dipole moment component along the principal $c$-axis is not identically zero. 
Table S15. Complete results of the bond distances and proton transfer parameters for conformers of the mono-, di-, and trihydrate of triflic acid across various levels of theory. ${ }^{a, b}$

\begin{tabular}{|c|c|c|c|c|c|}
\hline & $\begin{array}{c}\mathrm{M} 06-2 \mathrm{X} \\
6-311++\mathrm{G}(3 \mathrm{df}, 3 \mathrm{pd})\end{array}$ & $\begin{array}{c}\text { B3LYP-D3BJ } \\
\text { def2-TZVP }\end{array}$ & $\begin{array}{l}\text { B3LYP-D3BJ } \\
\text { jun-cc-pVDZ }\end{array}$ & $\begin{array}{l}\text { B2PLYP-D3BJ } \\
\text { jun-cc-pVTZ }\end{array}$ & $\begin{array}{l}\text { DSD-PBEP86-D3BJ } \\
\text { jun-cc-pVTZ }\end{array}$ \\
\hline $\begin{array}{c}\text { Free } \mathrm{CF}_{3} \mathrm{SO}_{3} \mathrm{H} \\
R(\mathrm{O}-\mathrm{H})\end{array}$ & 0.968 & 0.971 & 0.977 & 0.969 & 0.970 \\
\hline $\begin{array}{l}\text { Free } \mathrm{H}_{3} \mathrm{O}^{+} \\
R(\mathrm{O}-\mathrm{H})\end{array}$ & 1.019 & 1.043 & 1.037 & 1.030 & 1.027 \\
\hline & \multicolumn{5}{|c|}{$R(\mathrm{O} 2-\mathrm{H} 3)$} \\
\hline IA & 1.011 & 1.012 & 1.016 & 1.007 & 1.007 \\
\hline IB & 1.012 & 1.012 & 1.016 & 1.007 & 1.008 \\
\hline IIA & 1.057 & 1.052 & 1.055 & 1.040 & 1.040 \\
\hline IIB & 1.058 & 1.053 & 1.054 & 1.039 & 1.039 \\
\hline IIC & 1.056 & 1.051 & 1.054 & 1.039 & 1.039 \\
\hline IID & 1.051 & 1.048 & 1.049 & 1.036 & 1.036 \\
\hline IIIA & 1.517 & 1.547 & 1.544 & 1.542 & 1.537 \\
\hline IIIB & 1.519 & 1.548 & 1.546 & 1.541 & 1.537 \\
\hline IIIC & 1.086 & 1.076 & 1.076 & 1.055 & 1.056 \\
\hline IIID & 1.345 & 1.377 & 1.434 & 1.443 & 1.449 \\
\hline & \multicolumn{5}{|c|}{$R(\mathrm{H3} \cdots \mathrm{O} 10)$} \\
\hline IA & 1.580 & 1.611 & 1.614 & 1.621 & 1.615 \\
\hline IB & 1.577 & 1.609 & 1.610 & 1.618 & 1.611 \\
\hline IIA & 1.417 & 1.451 & 1.460 & 1.478 & 1.473 \\
\hline IIB & 1.416 & 1.450 & 1.463 & 1.481 & 1.476 \\
\hline IIC & 1.423 & 1.454 & 1.463 & 1.482 & 1.477 \\
\hline IID & 1.433 & 1.462 & 1.476 & 1.490 & 1.486 \\
\hline IIIA & 1.027 & 1.027 & 1.031 & 1.024 & 1.024 \\
\hline IIIB & 1.027 & 1.028 & 1.031 & 1.025 & 1.024 \\
\hline IIIC & 1.350 & 1.386 & 1.400 & 1.429 & 1.423 \\
\hline IIID & 1.091 & 1.088 & 1.069 & 1.057 & 1.053 \\
\hline \multicolumn{6}{|c|}{$\rho_{\mathrm{PT}}$} \\
\hline IA & -0.52 & -0.53 & -0.54 & -0.55 & -0.55 \\
\hline IB & -0.51 & -0.53 & -0.53 & -0.55 & -0.55 \\
\hline IIA & -0.31 & $\begin{array}{l}-0.33 \\
\end{array}$ & -0.34 & $\begin{array}{l}-0.38 \\
\end{array}$ & -0.38 \\
\hline IIB & -0.31 & -0.33 & -0.35 & -0.38 & -0.38 \\
\hline IIC & -0.32 & -0.33 & -0.35 & -0.38 & -0.38 \\
\hline IID & -0.33 & -0.34 & -0.37 & -0.39 & -0.39 \\
\hline IIIA & +0.54 & +0.59 & +0.57 & +0.58 & +0.57 \\
\hline IIIB & +0.54 & +0.59 & +0.57 & +0.58 & +0.57 \\
\hline IIIC & -0.21 & -0.26 & -0.27 & -0.32 & -0.32 \\
\hline IIID & +0.31 & +0.36 & +0.43 & +0.45 & +0.45 \\
\hline
\end{tabular}

(a) All distances in $\AA$.

(b) The proton transfer parameter $\left(\rho_{\mathrm{PT}}\right)$ is calculated as $\rho_{\mathrm{PT}}=\left(R(\mathrm{O} 2-\mathrm{H} 3)_{\text {complex }}-R(\mathrm{O}-\mathrm{H})_{\mathrm{CF}_{3} \mathrm{SO}_{3} \mathrm{H}}\right)$ $-\left(R(\mathrm{H} 3 \cdots \mathrm{O} 10)_{\text {complex }}-R(\mathrm{H}-\mathrm{O})_{\mathrm{H}_{3} \mathrm{O}^{+}}\right)$

(c) For calculations using the jun-cc-pVDZ or jun-cc-pVTZ basis sets, the jun-cc-pV(D+d)Z or jun-cc-pV(T+d)Z basis set was used on the sulfur atom, respectively. 
Table S16. Cartesian coordinates (in $\AA$ ) of the equilibrium minimum-energy structure of $\mathrm{CF}_{3} \mathrm{SO}_{3} \mathrm{H} \cdots \mathrm{H}_{2} \mathrm{O}$ structure (IA) resulting from B2PLYP-D3BJ/jun-cc-pVTZ and DSD-PBEP86D3BJ/jun-cc-pVTZ calculations (top). Cartesian coordinates of the equilibrium and vibrationally averaged structure calculated at the B3LYP-D3BJ/jun-cc-pVDZ level (bottom).

\begin{tabular}{lccc|ccc}
\hline & \multicolumn{2}{c}{ B2PLYP-D3BJ/jun-cc-pVTZ } & \multicolumn{2}{c}{ DSD-PBEP86-D3BJ/jun-cc-pVTZ } \\
\cline { 2 - 6 } & $\mathrm{X}$ & $\mathrm{Y}$ & $\mathrm{Z}$ & $\mathrm{X}$ & $\mathrm{Y}$ & $\mathrm{Z}$ \\
\hline $\mathrm{S}$ & 0.263886 & 0.778445 & 0.075407 & 0.256129 & 0.782857 & 0.075261 \\
$\mathrm{O}$ & 1.115289 & 0.357367 & -1.159350 & 1.108025 & 0.374084 & -1.157032 \\
$\mathrm{H}$ & 1.874286 & -0.231160 & -0.857338 & 1.862187 & -0.219931 & -0.852885 \\
$\mathrm{C}$ & -1.077599 & -0.495667 & -0.001903 & -1.063503 & -0.500905 & -0.001911 \\
$\mathrm{~F}$ & -1.745863 & -0.397166 & -1.144674 & -1.733323 & -0.408265 & -1.141178 \\
$\mathrm{~F}$ & -0.545365 & -1.714978 & 0.088586 & -0.515690 & -1.710741 & 0.084321 \\
$\mathrm{~F}$ & -1.912472 & -0.308126 & 1.014774 & -1.897064 & -0.326984 & 1.014721 \\
$\mathrm{O}$ & -0.349084 & 2.036400 & -0.180092 & -0.370412 & 2.031960 & -0.177440 \\
$\mathrm{O}$ & 0.993132 & 0.510777 & 1.285303 & 0.987016 & 0.520934 & 1.282405 \\
$\mathrm{O}$ & 2.976011 & -1.091126 & -0.036013 & 2.951077 & -1.089130 & -0.037139 \\
$\mathrm{H}$ & 3.915025 & -0.926513 & -0.160426 & 3.890051 & -0.921159 & -0.158536 \\
$\mathrm{H}$ & 2.760087 & -0.791778 & 0.857794 & 2.735745 & -0.788804 & 0.856657 \\
\hline
\end{tabular}

\begin{tabular}{cccc|ccc}
\hline & \multicolumn{5}{c}{ B3LYP-D3BJ/jun-cc-pVDZ } \\
\cline { 2 - 7 } & $\mathrm{X}$ & Equilibrium & $\mathrm{Z}$ & $\mathrm{X}$ & $\mathrm{Y}$ & $\mathrm{Z}$ \\
\hline $\mathrm{S}$ & 0.271088 & 0.785299 & 0.079376 & 0.271537 & 0.783599 & 0.081819 \\
$\mathrm{O}$ & 1.132013 & 0.354681 & -1.177395 & 1.136613 & 0.338489 & -1.172405 \\
$\mathrm{H}$ & 1.891249 & -0.242138 & -0.862547 & 1.910643 & -0.246264 & -0.855978 \\
$\mathrm{C}$ & -1.089804 & -0.495618 & -0.002854 & -1.101840 & -0.495478 & -0.003223 \\
$\mathrm{~F}$ & -1.768108 & -0.387075 & -1.147338 & -1.780720 & -0.383059 & -1.149928 \\
$\mathrm{~F}$ & -0.557655 & -1.724380 & 0.077205 & -0.577282 & -1.730617 & 0.076417 \\
$\mathrm{~F}$ & -1.924484 & -0.313151 & 1.025307 & -1.937871 & -0.309641 & 1.026458 \\
$\mathrm{O}$ & -0.338894 & 2.059434 & -0.178067 & -0.331657 & 2.062518 & -0.183376 \\
$\mathrm{O}$ & 1.009228 & 0.502664 & 1.298142 & 1.002028 & 0.503517 & 1.308502 \\
$\mathrm{O}$ & 2.978833 & -1.103664 & -0.037127 & 3.028905 & -1.084570 & -0.046794 \\
$\mathrm{H}$ & 3.935624 & -1.008945 & -0.156109 & 3.949802 & -1.050881 & -0.187411 \\
$\mathrm{H}$ & 2.789434 & -0.829846 & 0.878496 & 2.876746 & -0.864342 & 0.866128 \\
\hline
\end{tabular}


Table S17. Cartesian coordinates (in $\AA$ ) of the minimum-energy structure of $\mathrm{CF}_{3} \mathrm{SO}_{3} \mathrm{H} \cdots \mathrm{H}_{2} \mathrm{O}$ structure (IB) resulting from B2PLYP-D3BJ/jun-cc-pVTZ and DSD-PBEP86-D3BJ/jun-cc-pVTZ calculations (top). Cartesian coordinates of the equilibrium and vibrationally averaged structure calculated at the B3LYP-D3BJ/jun-cc-pVDZ level (bottom).

\begin{tabular}{lccc|ccc}
\hline & \multicolumn{3}{c|}{ B2PLYP-D3BJ/jun-cc-pVTZ } & \multicolumn{2}{c}{ DSD-PBEP86-D3BJ/jun-cc-pVTZ } \\
\cline { 2 - 6 } & $\mathrm{X}$ & $\mathrm{Y}$ & $\mathrm{Z}$ & $\mathrm{X}$ & $\mathrm{Y}$ & $\mathrm{Z}$ \\
\hline $\mathrm{S}$ & 0.255193 & 0.794429 & 0.074814 & 0.246873 & 0.799466 & 0.074599 \\
$\mathrm{O}$ & 1.115112 & 0.382663 & -1.155961 & 1.107856 & 0.400704 & -1.153423 \\
$\mathrm{H}$ & 1.863885 & -0.219972 & -0.854358 & 1.851872 & -0.207230 & -0.849588 \\
$\mathrm{C}$ & -1.062199 & -0.504326 & -0.001273 & -1.047046 & -0.510077 & -0.001249 \\
$\mathrm{~F}$ & -1.735217 & -0.422947 & -1.141891 & -1.720835 & -0.436261 & -1.138818 \\
$\mathrm{~F}$ & -0.501870 & -1.715915 & 0.085556 & -0.470112 & -1.711241 & 0.082458 \\
$\mathrm{~F}$ & -1.898171 & -0.341548 & 1.017691 & -1.882061 & -0.361235 & 1.017072 \\
$\mathrm{O}$ & -0.380489 & 2.039099 & -0.187799 & -0.403663 & 2.034196 & -0.185712 \\
$\mathrm{O}$ & 0.982218 & 0.541008 & 1.288484 & 0.975387 & 0.553058 & 1.285804 \\
$\mathrm{O}$ & 3.000808 & -1.036107 & -0.042925 & 2.975036 & -1.034561 & -0.043913 \\
$\mathrm{H}$ & 3.026428 & -1.996774 & -0.075307 & 2.973671 & -1.995789 & -0.075238 \\
$\mathrm{H}$ & 2.742214 & -0.800248 & 0.858431 & 2.717432 & -0.795591 & 0.857094 \\
\hline
\end{tabular}

\begin{tabular}{cccc|ccc}
\hline & \multicolumn{5}{c}{ B3LYP-D3BJ/jun-cc-pVDZ } \\
\cline { 2 - 7 } & $\mathrm{X}$ & Equilibrium & $\mathrm{Z}$ & $\mathrm{X}$ & $\mathrm{Y}$ & $\mathrm{Z}$ \\
\hline $\mathrm{S}$ & 0.262250 & 0.802379 & 0.078770 & 0.262001 & 0.804865 & 0.081022 \\
$\mathrm{O}$ & 1.132217 & 0.380910 & -1.173235 & 1.138407 & 0.372052 & -1.168236 \\
$\mathrm{H}$ & 1.880386 & -0.230882 & -0.858429 & 1.898539 & -0.232045 & -0.851551 \\
$\mathrm{C}$ & -1.073650 & -0.504249 & -0.002296 & -1.081668 & -0.505306 & -0.002408 \\
$\mathrm{~F}$ & -1.760236 & -0.410889 & -1.142396 & -1.769874 & -0.409100 & -1.144367 \\
$\mathrm{~F}$ & -0.511231 & -1.725241 & 0.068872 & -0.522244 & -1.730521 & 0.067018 \\
$\mathrm{~F}$ & -1.906586 & -0.351122 & 1.030899 & -1.916076 & -0.352025 & 1.032897 \\
$\mathrm{O}$ & -0.371002 & 2.062970 & -0.186285 & -0.369126 & 2.067914 & -0.192669 \\
$\mathrm{O}$ & 0.997441 & 0.534002 & 1.301828 & 0.990751 & 0.542649 & 1.312011 \\
$\mathrm{O}$ & 2.997989 & -1.054842 & -0.043829 & 3.026336 & -1.059189 & -0.052356 \\
$\mathrm{H}$ & 3.078940 & -2.019903 & -0.076097 & 3.169211 & -1.978624 & -0.108986 \\
$\mathrm{H}$ & 2.773488 & -0.832838 & 0.877710 & 2.864912 & -0.862558 & 0.863753 \\
\hline
\end{tabular}


Table S18. Cartesian coordinates (in $\AA$ ) of the $\mathrm{CF}_{3} \mathrm{SO}_{3} \mathrm{H} \cdots \mathrm{H}_{2} \mathrm{O}$ transition state structure TS(I) resulting from B2PLYP-D3BJ/jun-cc-pVTZ and DSD-PBEP86-D3BJ/jun-cc-pVTZ calculations.

\begin{tabular}{lccc|ccc} 
& \multicolumn{2}{c}{ B2PLYP-D3BJ/jun-cc-pVTZ } & \multicolumn{2}{c}{ DSD-PBEP86-D3BJ/jun-cc-pVTZ } \\
\cline { 2 - 6 } & $\mathrm{X}$ & $\mathrm{Y}$ & $\mathrm{Z}$ & $\mathrm{X}$ & $\mathrm{Y}$ & $\mathrm{Z}$ \\
\hline $\mathrm{S}$ & 0.254961 & 0.787935 & 0.078979 & 0.247083 & 0.791844 & 0.079074 \\
$\mathrm{O}$ & 1.103338 & 0.390966 & -1.168710 & 1.094374 & 0.408830 & -1.167702 \\
$\mathrm{H}$ & 1.856347 & -0.200836 & -0.887619 & 1.843441 & -0.187344 & -0.886313 \\
$\mathrm{C}$ & -1.066310 & -0.507180 & -0.001317 & -1.052279 & -0.512535 & -0.001295 \\
$\mathrm{~F}$ & -1.744181 & -0.410738 & -1.138866 & -1.732560 & -0.420760 & -1.134696 \\
$\mathrm{~F}$ & -0.515776 & -1.720069 & 0.075277 & -0.486713 & -1.715840 & 0.069481 \\
$\mathrm{~F}$ & -1.897946 & -0.342298 & 1.022242 & -1.881404 & -0.361801 & 1.022976 \\
$\mathrm{O}$ & -0.381286 & 2.035409 & -0.171216 & -0.403052 & 2.030360 & -0.166358 \\
$\mathrm{O}$ & 0.993315 & 0.525750 & 1.281958 & 0.988714 & 0.534422 & 1.277975 \\
$\mathrm{O}$ & 2.982005 & -1.039197 & -0.041470 & 2.958511 & -1.035270 & -0.042580 \\
$\mathrm{H}$ & 3.725355 & -1.636708 & -0.140883 & 3.697309 & -1.638677 & -0.140528 \\
$\mathrm{H}$ & 2.855811 & -0.859188 & 0.896233 & 2.834451 & -0.857450 & 0.895855 \\
\hline
\end{tabular}

Table S19. Cartesian coordinates (in $\AA$ ) of the $\mathrm{CF}_{3} \mathrm{SO}_{3} \mathrm{H} \cdots \mathrm{H}_{2} \mathrm{O}$ transition state structure TS(II) resulting from B2PLYP-D3BJ/jun-cc-pVTZ and DSD-PBEP86-D3BJ/jun-cc-pVTZ calculations.

\begin{tabular}{lccc|ccc}
\hline & \multicolumn{2}{c|}{ B2PLYP-D3BJ/jun-cc-pVTZ } & \multicolumn{2}{c}{ DSD-PBEP86-D3BJ/jun-cc-pVTZ } \\
\cline { 2 - 6 } & $\mathrm{X}$ & $\mathrm{Y}$ & $\mathrm{Z}$ & $\mathrm{X}$ & $\mathrm{Y}$ & $\mathrm{Z}$ \\
\hline $\mathrm{S}$ & 0.181012 & 0.855791 & 0.077193 & 0.177233 & 0.856475 & 0.077216 \\
$\mathrm{O}$ & 1.142155 & 0.469010 & -1.086908 & 1.129354 & 0.485055 & -1.092655 \\
$\mathrm{H}$ & 1.878159 & -0.123581 & -0.742153 & 1.862299 & -0.113857 & -0.751052 \\
$\mathrm{C}$ & -1.002427 & -0.565666 & -0.004150 & -0.989207 & -0.567350 & -0.004008 \\
$\mathrm{~F}$ & -1.616723 & -0.606285 & -1.178952 & -1.610355 & -0.605609 & -1.172171 \\
$\mathrm{~F}$ & -0.329451 & -1.712568 & 0.170504 & -0.304824 & -1.706382 & 0.157370 \\
$\mathrm{~F}$ & -1.904878 & -0.446996 & 0.962490 & -1.883177 & -0.463851 & 0.968459 \\
$\mathrm{O}$ & -0.545971 & 2.020970 & -0.294302 & -0.560282 & 2.016675 & -0.279561 \\
$\mathrm{O}$ & 0.850799 & 0.726977 & 1.338152 & 0.855669 & 0.722658 & 1.330418 \\
$\mathrm{O}$ & 3.007505 & -1.075167 & -0.070740 & 2.972075 & -1.072853 & -0.071615 \\
$\mathrm{H}$ & 3.643504 & -0.535685 & 0.410990 & 3.600443 & -0.532783 & 0.419800 \\
$\mathrm{H}$ & 2.571495 & -1.607461 & 0.604121 & 2.514810 & -1.588012 & 0.602924 \\
\hline
\end{tabular}


Table S20. Cartesian coordinates (in $\AA$ ) of the $\mathrm{CF}_{3} \mathrm{SO}_{3} \mathrm{H} \cdots \mathrm{H}_{2} \mathrm{O}$ transition state structure TS(III) resulting from B2PLYP-D3BJ/jun-cc-pVTZ and DSD-PBEP86-D3BJ/jun-cc-pVTZ calculations.

\begin{tabular}{lccc|ccc}
\hline & \multicolumn{3}{c|}{ B2PLYP-D3BJ/jun-cc-pVTZ } & \multicolumn{2}{c}{ DSD-PBEP86-D3BJ/jun-cc-pVTZ } \\
\cline { 2 - 6 } & $\mathrm{X}$ & $\mathrm{Y}$ & $\mathrm{Z}$ & $\mathrm{X}$ & $\mathrm{Y}$ & $\mathrm{Z}$ \\
\hline $\mathrm{S}$ & 0.379699 & 0.689621 & -0.000000 & 0.374252 & 0.694051 & 0.000000 \\
$\mathrm{O}$ & 1.109511 & 0.235070 & -1.211660 & 1.104247 & 0.245687 & -1.209708 \\
$\mathrm{H}$ & 2.196068 & -0.593020 & -0.763555 & 2.178921 & -0.588614 & -0.760579 \\
$\mathrm{C}$ & -1.123046 & -0.386921 & -0.000000 & -1.111981 & -0.390495 & 0.000000 \\
$\mathrm{~F}$ & -1.857163 & -0.158691 & -1.083288 & -1.846400 & -0.169842 & -1.081268 \\
$\mathrm{~F}$ & -0.748266 & -1.675939 & 0.000000 & -0.724580 & -1.672974 & -0.000000 \\
$\mathrm{~F}$ & -1.857163 & -0.158691 & 1.083288 & -1.846400 & -0.169842 & 1.081268 \\
$\mathrm{O}$ & -0.087259 & 2.036104 & 0.000000 & -0.102706 & 2.034397 & -0.000000 \\
$\mathrm{O}$ & 1.109511 & 0.235070 & 1.211660 & 1.104247 & 0.245687 & 1.209708 \\
$\mathrm{O}$ & 2.805594 & -1.026900 & 0.000000 & 2.785380 & -1.030159 & 0.000000 \\
$\mathrm{H}$ & 2.698692 & -1.986737 & -0.000000 & 2.654814 & -1.987244 & 0.000000 \\
$\mathrm{H}$ & 2.196068 & -0.593020 & 0.763555 & 2.178921 & -0.588614 & 0.760579 \\
\hline
\end{tabular}


Table S21. Cartesian coordinates (in $\AA$ ) of the minimum-energy structure of $\mathrm{CF}_{3} \mathrm{SO}_{3} \mathrm{H} \cdots\left(\mathrm{H}_{2} \mathrm{O}\right)_{2}$ structure (IIA) resulting from B2PLYP-D3BJ/jun-cc-pVTZ and DSD-PBEP86-D3BJ/jun-cc-pVTZ calculations (top). Cartesian coordinates of the equilibrium and vibrationally averaged structure calculated at the B3LYP-D3BJ/jun-cc-pVDZ level (bottom).

\begin{tabular}{cccc|ccc}
\hline & \multicolumn{3}{c|}{ B2PLYP-D3BJ/jun-cc-pVTZ } & \multicolumn{2}{c}{ DSD-PBEP86-D3BJ/jun-cc-pVTZ } \\
\cline { 2 - 6 } & $\mathrm{X}$ & $\mathrm{Y}$ & $\mathrm{Z}$ & $\mathrm{X}$ & $\mathrm{Y}$ & $\mathrm{Z}$ \\
\hline $\mathrm{S}$ & 0.223886 & 0.886809 & 0.030998 & 0.228795 & 0.885652 & 0.040764 \\
$\mathrm{O}$ & -0.427949 & 0.641070 & 1.402897 & -0.411633 & 0.631192 & 1.411720 \\
$\mathrm{H}$ & -1.291494 & 0.063510 & 1.361330 & -1.270861 & 0.047464 & 1.362181 \\
$\mathrm{C}$ & 1.260653 & -0.637031 & -0.136726 & 1.249537 & -0.635904 & -0.147509 \\
$\mathrm{~F}$ & 2.167441 & -0.694356 & 0.831209 & 2.161680 & -0.706935 & 0.810756 \\
$\mathrm{~F}$ & 0.478990 & -1.720886 & -0.060169 & 0.462117 & -1.712491 & -0.073112 \\
$\mathrm{~F}$ & 1.873603 & -0.630367 & -1.314896 & 1.851393 & -0.623890 & -1.328287 \\
$\mathrm{O}$ & 1.120053 & 1.988914 & 0.114748 & 1.130843 & 1.979768 & 0.126782 \\
$\mathrm{O}$ & -0.744644 & 0.797665 & -1.031542 & -0.746975 & 0.809905 & -1.012521 \\
$\mathrm{O}$ & -2.547318 & -0.716486 & 1.341969 & -2.513765 & -0.742322 & 1.331833 \\
$\mathrm{H}$ & -2.447761 & -1.659014 & 1.501780 & -2.393659 & -1.688880 & 1.449582 \\
$\mathrm{H}$ & -2.976004 & -0.618524 & 0.457862 & -2.954012 & -0.621006 & 0.456753 \\
$\mathrm{O}$ & -3.326626 & -0.279139 & -1.171716 & -3.337608 & -0.249532 & -1.157498 \\
$\mathrm{H}$ & -4.043858 & 0.297358 & -1.446678 & -4.055476 & 0.339665 & -1.402727 \\
$\mathrm{H}$ & -2.502874 & 0.183195 & -1.394362 & -2.517424 & 0.217126 & -1.382991 \\
\hline
\end{tabular}

\begin{tabular}{cccc|ccc}
\hline & \multicolumn{5}{c}{ B3LYP-D3BJ/jun-cc-pVDZ } \\
\cline { 2 - 6 } & $\mathrm{X}$ & Equilibrium & \multicolumn{3}{c}{ Ground vibrational state } \\
& $\mathrm{Y}$ & $\mathrm{Y}$ & $\mathrm{X}$ & $\mathrm{Y}$ & $\mathrm{Z}$ \\
\hline $\mathrm{S}$ & 0.252528 & 0.915314 & -0.059621 & 0.246973 & 0.911548 & -0.062876 \\
$\mathrm{O}$ & -0.414826 & 0.845835 & 1.348532 & -0.427606 & 0.831504 & 1.340156 \\
$\mathrm{H}$ & -1.303500 & 0.277477 & 1.363390 & -1.343716 & 0.254822 & 1.364230 \\
$\mathrm{C}$ & 1.217910 & -0.686042 & -0.067396 & 1.231156 & -0.687086 & -0.065195 \\
$\mathrm{~F}$ & 2.139585 & -0.688687 & 0.898263 & 2.143613 & -0.688448 & 0.912251 \\
$\mathrm{~F}$ & 0.374976 & -1.717760 & 0.135754 & 0.393218 & -1.729745 & 0.125253 \\
$\mathrm{~F}$ & 1.816376 & -0.845138 & -1.251044 & 1.846355 & -0.841192 & -1.243433 \\
$\mathrm{O}$ & 1.210829 & 1.984821 & -0.089902 & 1.198379 & 1.990926 & -0.099373 \\
$\mathrm{O}$ & -0.735157 & 0.759127 & -1.118934 & -0.733991 & 0.743293 & -1.131698 \\
$\mathrm{O}$ & -2.552332 & -0.477606 & 1.406944 & -2.553512 & -0.476260 & 1.408258 \\
$\mathrm{H}$ & -2.492679 & -1.389800 & 1.727319 & -2.499073 & -1.359070 & 1.728835 \\
$\mathrm{H}$ & -2.964631 & -0.522009 & 0.499012 & -2.976692 & -0.510079 & 0.505967 \\
$\mathrm{O}$ & -3.242559 & -0.461724 & -1.154506 & -3.271449 & -0.422647 & -1.134639 \\
$\mathrm{H}$ & -3.969053 & 0.006557 & -1.590167 & -3.979505 & -0.006536 & -1.574973 \\
$\mathrm{H}$ & -2.421329 & -0.010268 & -1.437988 & -2.459500 & 0.002098 & -1.444995 \\
\hline & & & & & \\
\hline
\end{tabular}


Table S22. Cartesian coordinates (in $\AA$ ) of the minimum-energy structure of $\mathrm{CF}_{3} \mathrm{SO}_{3} \mathrm{H} \cdots\left(\mathrm{H}_{2} \mathrm{O}\right)_{2}$ structure (IIB) resulting from B2PLYP-D3BJ/jun-cc-pVTZ and DSD-PBEP86-D3BJ/jun-cc-pVTZ calculations (top). Cartesian coordinates of the equilibrium and vibrationally averaged structure calculated at the B3LYP-D3BJ/jun-cc-pVDZ level (bottom).

\begin{tabular}{cccc|ccc}
\hline & \multicolumn{3}{c|}{ B2PLYP-D3BJ/jun-cc-pVTZ } & \multicolumn{2}{c}{ DSD-PBEP86-D3BJ/jun-cc-pVTZ } \\
\cline { 2 - 6 } & $\mathrm{X}$ & $\mathrm{Y}$ & $\mathrm{Z}$ & $\mathrm{X}$ & $\mathrm{Y}$ & $\mathrm{Z}$ \\
\hline $\mathrm{S}$ & 0.278663 & 0.871502 & -0.266747 & 0.303607 & 0.871507 & -0.289717 \\
$\mathrm{O}$ & -0.399321 & 1.153529 & 1.085257 & -0.368576 & 1.214432 & 1.046135 \\
$\mathrm{H}$ & -1.325516 & 0.696835 & 1.199523 & -1.303903 & 0.777229 & 1.164482 \\
$\mathrm{C}$ & 1.183563 & -0.695376 & 0.121087 & 1.140564 & -0.710236 & 0.145269 \\
$\mathrm{~F}$ & 2.056004 & -0.503850 & 1.102307 & 2.011527 & -0.526876 & 1.125527 \\
$\mathrm{~F}$ & 0.309136 & -1.635468 & 0.494270 & 0.227682 & -1.601900 & 0.536757 \\
$\mathrm{~F}$ & 1.826996 & -1.112989 & -0.965041 & 1.771988 & -1.182586 & -0.922011 \\
$\mathrm{O}$ & 1.264159 & 1.864133 & -0.527812 & 1.326057 & 1.816704 & -0.571088 \\
$\mathrm{O}$ & -0.689339 & 0.506263 & -1.269642 & -0.670649 & 0.514468 & -1.285770 \\
$\mathrm{O}$ & -2.637761 & 0.042838 & 1.411033 & -2.621077 & 0.148801 & 1.382874 \\
$\mathrm{H}$ & -3.336213 & 0.626432 & 1.719676 & -3.322828 & 0.761137 & 1.620931 \\
$\mathrm{H}$ & -2.949332 & -0.356623 & 0.563253 & -2.911930 & -0.306875 & 0.556746 \\
$\mathrm{O}$ & -3.138339 & -0.843952 & -1.054568 & -3.082443 & -0.899160 & -1.026805 \\
$\mathrm{H}$ & -3.148612 & -1.767607 & -1.317751 & -3.055627 & -1.838359 & -1.226811 \\
$\mathrm{H}$ & -2.312975 & -0.470788 & -1.403516 & -2.265681 & -0.524491 & -1.393243 \\
\hline
\end{tabular}

\begin{tabular}{lccc|ccc}
\hline & \multicolumn{5}{c}{ B3LYP-D3BJ/jun-cc-pVDZ } \\
\cline { 2 - 6 } & $\mathrm{X}$ & Equilibrium & $\mathrm{Z}$ & $\mathrm{X}$ & $\mathrm{Y}$ & $\mathrm{Z}$ \\
\hline $\mathrm{S}$ & 0.289160 & 0.888866 & -0.273997 & 0.285220 & 0.889838 & -0.269211 \\
$\mathrm{O}$ & -0.402939 & 1.195586 & 1.089805 & -0.410960 & 1.177382 & 1.095531 \\
$\mathrm{H}$ & -1.342284 & 0.730194 & 1.201297 & -1.386370 & 0.723465 & 1.208443 \\
$\mathrm{C}$ & 1.165610 & -0.711889 & 0.131074 & 1.173601 & -0.715086 & 0.125792 \\
$\mathrm{~F}$ & 2.042753 & -0.538641 & 1.121083 & 2.044119 & -0.550543 & 1.125964 \\
$\mathrm{~F}$ & 0.260104 & -1.634501 & 0.505899 & 0.269998 & -1.650547 & 0.484701 \\
$\mathrm{~F}$ & 1.806876 & -1.155552 & -0.955903 & 1.827259 & -1.145793 & -0.961758 \\
$\mathrm{O}$ & 1.304471 & 1.870790 & -0.534984 & 1.299361 & 1.876770 & -0.532442 \\
$\mathrm{O}$ & -0.689883 & 0.528922 & -1.291466 & -0.691271 & 0.528827 & -1.294210 \\
$\mathrm{O}$ & -2.636106 & 0.074559 & 1.390320 & -2.659224 & 0.126471 & 1.380403 \\
$\mathrm{H}$ & -3.366983 & 0.641493 & 1.677504 & -3.359194 & 0.693092 & 1.649512 \\
$\mathrm{H}$ & -2.932923 & -0.367393 & 0.545711 & -2.949680 & -0.339986 & 0.548029 \\
$\mathrm{O}$ & -3.089646 & -0.878344 & -1.043662 & -3.081568 & -0.899632 & -1.019040 \\
$\mathrm{H}$ & -3.091190 & -1.801411 & -1.335298 & -3.121122 & -1.783982 & -1.307105 \\
$\mathrm{H}$ & -2.277952 & -0.479257 & -1.418717 & -2.292500 & -0.509361 & -1.420407 \\
\hline & & & & & \\
\hline
\end{tabular}


Table S23. Cartesian coordinates (in $\AA$ ) of the minimum-energy structure of $\mathrm{CF}_{3} \mathrm{SO}_{3} \mathrm{H} \cdots\left(\mathrm{H}_{2} \mathrm{O}\right)_{2}$ structure (IIC) resulting from B2PLYP-D3BJ/jun-cc-pVTZ and DSD-PBEP86-D3BJ/jun-cc-pVTZ calculations (top). Cartesian coordinates of the equilibrium and vibrationally averaged structure calculated at the B3LYP-D3BJ/jun-cc-pVDZ level (bottom).

\begin{tabular}{cccc|ccc}
\hline & \multicolumn{3}{c|}{ B2PLYP-D3BJ/jun-cc-pVTZ } & \multicolumn{2}{c}{ DSD-PBEP86-D3BJ/jun-cc-pVTZ } \\
\cline { 2 - 6 } & $\mathrm{X}$ & $\mathrm{Y}$ & $\mathrm{Z}$ & $\mathrm{X}$ & $\mathrm{Y}$ & $\mathrm{Z}$ \\
\hline $\mathrm{S}$ & -0.195024 & 0.863261 & -0.138595 & 0.193271 & 0.857328 & 0.152415 \\
$\mathrm{O}$ & 0.458037 & 0.410648 & -1.456202 & -0.449235 & 0.382007 & 1.462289 \\
$\mathrm{H}$ & 1.288310 & -0.199305 & -1.324303 & -1.269600 & -0.237848 & 1.313603 \\
$\mathrm{C}$ & -1.295351 & -0.582408 & 0.214342 & 1.291613 & -0.571935 & -0.226396 \\
$\mathrm{~F}$ & -2.197231 & -0.731537 & -0.747877 & 2.197360 & -0.731465 & 0.726759 \\
$\mathrm{~F}$ & -0.558289 & -1.698691 & 0.290211 & 0.558613 & -1.686905 & -0.313621 \\
$\mathrm{~F}$ & -1.915167 & -0.398139 & 1.374623 & 1.902622 & -0.369362 & -1.385152 \\
$\mathrm{O}$ & -1.045092 & 1.978090 & -0.381135 & 1.038867 & 1.969902 & 0.407136 \\
$\mathrm{O}$ & 0.757971 & 0.874632 & 0.941148 & -0.763612 & 0.879267 & -0.920220 \\
$\mathrm{O}$ & 2.495935 & -1.046641 & -1.180050 & -2.456337 & -1.100200 & 1.142993 \\
$\mathrm{H}$ & 2.320704 & -1.990117 & -1.128352 & -2.250822 & -2.031499 & 1.022488 \\
$\mathrm{H}$ & 2.968981 & -0.794698 & -0.352953 & -2.947185 & -0.812292 & 0.338759 \\
$\mathrm{O}$ & 3.426245 & 0.032741 & 1.078401 & -3.445339 & 0.073135 & -1.043107 \\
$\mathrm{H}$ & 3.800163 & -0.295344 & 1.899148 & -3.836343 & -0.224516 & -1.867647 \\
$\mathrm{H}$ & 2.577053 & 0.444691 & 1.302067 & -2.599988 & 0.488761 & -1.273243 \\
\hline
\end{tabular}

\begin{tabular}{cccc|ccc}
\hline & \multicolumn{5}{c}{ B3LYP-D3BJ/jun-cc-pVDZ } \\
\cline { 2 - 7 } & $\mathrm{X}$ & Equilibrium & $\mathrm{Z}$ & $\mathrm{X}$ & $\mathrm{Y}$ & $\mathrm{Z}$ \\
\hline $\mathrm{S}$ & -0.208518 & 0.892631 & -0.092343 & -0.200714 & 0.884744 & -0.084021 \\
$\mathrm{O}$ & 0.458311 & 0.530479 & -1.455197 & 0.478104 & 0.511506 & -1.438062 \\
$\mathrm{H}$ & 1.303730 & -0.090579 & -1.355509 & 1.346076 & -0.122121 & -1.343667 \\
$\mathrm{C}$ & -1.276583 & -0.617048 & 0.183650 & -1.293348 & -0.617288 & 0.179081 \\
$\mathrm{~F}$ & -2.191343 & -0.735083 & -0.780974 & -2.204696 & -0.722963 & -0.793168 \\
$\mathrm{~F}$ & -0.501785 & -1.721366 & 0.181744 & -0.530897 & -1.733452 & 0.178502 \\
$\mathrm{~F}$ & -1.890317 & -0.525758 & 1.366883 & -1.916257 & -0.525039 & 1.359817 \\
$\mathrm{O}$ & -1.096766 & 2.007455 & -0.268440 & -1.077559 & 2.012220 & -0.260997 \\
$\mathrm{O}$ & 0.759987 & 0.861396 & 0.994730 & 0.754045 & 0.841076 & 1.018921 \\
$\mathrm{O}$ & 2.495962 & -0.932268 & -1.249251 & 2.506951 & -0.941213 & -1.248880 \\
$\mathrm{H}$ & 2.346611 & -1.888275 & -1.287840 & 2.368212 & -1.867540 & -1.268193 \\
$\mathrm{H}$ & 2.971393 & -0.751925 & -0.393068 & 3.007209 & -0.733294 & -0.418645 \\
$\mathrm{O}$ & 3.385884 & -0.070798 & 1.098814 & 3.396957 & -0.034500 & 1.066802 \\
$\mathrm{H}$ & 3.771912 & -0.416248 & 1.915278 & 3.997368 & -0.099823 & 1.828278 \\
$\mathrm{H}$ & 2.545496 & 0.363253 & 1.348839 & 2.586996 & 0.391736 & 1.372658 \\
\hline
\end{tabular}


Table S24. Cartesian coordinates (in $\AA$ ) of the minimum-energy structure of $\mathrm{CF}_{3} \mathrm{SO}_{3} \mathrm{H} \cdots\left(\mathrm{H}_{2} \mathrm{O}\right)_{2}$ structure (IID) resulting from B2PLYP-D3BJ/jun-cc-pVTZ and DSD-PBEP86-D3BJ/jun-cc-pVTZ calculations (top). Cartesian coordinates of the equilibrium and vibrationally averaged structure calculated at the B3LYP-D3BJ/jun-cc-pVDZ level (bottom).

\begin{tabular}{cccc|ccc}
\hline & \multicolumn{3}{c|}{ B2PLYP-D3BJ/jun-cc-pVTZ } & \multicolumn{2}{c}{ DSD-PBEP86-D3BJ/jun-cc-pVTZ } \\
\cline { 2 - 6 } & $\mathrm{X}$ & $\mathrm{Y}$ & $\mathrm{Z}$ & $\mathrm{X}$ & $\mathrm{Y}$ & $\mathrm{Z}$ \\
\hline $\mathrm{S}$ & -0.253208 & 0.873643 & 0.197073 & -0.283866 & 0.869274 & 0.259111 \\
$\mathrm{O}$ & 0.412200 & 1.022714 & -1.183794 & 0.378873 & 1.147543 & -1.098093 \\
$\mathrm{H}$ & 1.317739 & 0.527846 & -1.271243 & 1.298521 & 0.684753 & -1.210258 \\
$\mathrm{C}$ & -1.226959 & -0.680244 & -0.052933 & -1.175270 & -0.699628 & -0.109854 \\
$\mathrm{~F}$ & -2.115097 & -0.520668 & -1.027083 & -2.062573 & -0.513702 & -1.076170 \\
$\mathrm{~F}$ & -0.402614 & -1.678702 & -0.374119 & -0.300794 & -1.627494 & -0.494224 \\
$\mathrm{~F}$ & -1.861425 & -0.985980 & 1.074663 & -1.798379 & -1.117268 & 0.984508 \\
$\mathrm{O}$ & -1.192315 & 1.924939 & 0.392387 & -1.271095 & 1.856675 & 0.521633 \\
$\mathrm{O}$ & 0.719419 & 0.552120 & 1.210115 & 0.696440 & 0.520143 & 1.251862 \\
$\mathrm{O}$ & 2.617560 & -0.180434 & -1.445475 & 2.612709 & 0.024792 & -1.424974 \\
$\mathrm{H}$ & 3.287014 & 0.293609 & -1.945361 & 3.292042 & 0.585548 & -1.808792 \\
$\mathrm{H}$ & 3.008918 & -0.407090 & -0.569605 & 2.970897 & -0.320260 & -0.574359 \\
$\mathrm{O}$ & 3.195867 & -0.748156 & 1.102810 & 3.112692 & -0.878124 & 1.042584 \\
$\mathrm{H}$ & 3.897904 & -0.461032 & 1.691220 & 3.815657 & -0.704131 & 1.672909 \\
$\mathrm{H}$ & 2.380942 & -0.328942 & 1.422043 & 2.315525 & -0.458439 & 1.402694 \\
\hline
\end{tabular}

\begin{tabular}{lccc|ccc}
\hline & \multicolumn{5}{c}{ B3LYP-D3BJ/jun-cc-pVDZ } \\
\cline { 2 - 7 } & $\mathrm{X}$ & Equilibrium & $\mathrm{Z}$ & $\mathrm{X}$ & $\mathrm{Y}$ & $\mathrm{Z}$ \\
\hline $\mathrm{S}$ & 0.271861 & 0.889336 & -0.232650 & 0.265495 & 0.880138 & -0.232063 \\
$\mathrm{O}$ & -0.409680 & 1.115381 & 1.154593 & -0.421748 & 1.090556 & 1.154688 \\
$\mathrm{H}$ & -1.330352 & 0.621982 & 1.253071 & -1.370333 & 0.602839 & 1.258156 \\
$\mathrm{C}$ & 1.196924 & -0.703870 & 0.086615 & 1.218334 & -0.705448 & 0.087535 \\
$\mathrm{~F}$ & 2.093385 & -0.540965 & 1.062406 & 2.108709 & -0.535840 & 1.070593 \\
$\mathrm{~F}$ & 0.327776 & -1.661979 & 0.445315 & 0.361984 & -1.681786 & 0.438557 \\
$\mathrm{~F}$ & 1.825159 & -1.082441 & -1.031784 & 1.860789 & -1.072318 & -1.029521 \\
$\mathrm{O}$ & 1.254839 & 1.912253 & -0.458828 & 1.237919 & 1.916196 & -0.462830 \\
$\mathrm{O}$ & -0.714500 & 0.555461 & -1.251723 & -0.714262 & 0.530800 & -1.256933 \\
$\mathrm{O}$ & -2.621141 & -0.072916 & 1.428136 & -2.643774 & -0.042710 & 1.424962 \\
$\mathrm{H}$ & -3.326799 & 0.410273 & 1.881428 & -3.311506 & 0.411174 & 1.889813 \\
$\mathrm{H}$ & -2.994041 & -0.385372 & 0.559035 & -3.025219 & -0.361321 & 0.566810 \\
$\mathrm{O}$ & -3.125937 & -0.830178 & -1.071614 & -3.172160 & -0.784994 & -1.065679 \\
$\mathrm{H}$ & -3.820142 & -0.668902 & -1.725507 & -3.822134 & -0.749772 & -1.723898 \\
$\mathrm{H}$ & -2.315001 & -0.411379 & -1.425448 & -2.366399 & -0.413489 & -1.446591 \\
\hline & & & & & \\
\hline
\end{tabular}


Table S25. Cartesian coordinates (in $\AA$ ) of the minimum-energy structure of $\mathrm{CF}_{3} \mathrm{SO}_{3} \mathrm{H} \cdots\left(\mathrm{H}_{2} \mathrm{O}\right)_{3}$ structure (IIIA) resulting from B2PLYP-D3BJ/jun-cc-pVTZ and DSD-PBEP86-D3BJ/jun-cc-pVTZ calculations (top). Cartesian coordinates of the equilibrium and vibrationally averaged structure calculated at the B3LYP-D3BJ/jun-cc-pVDZ level (bottom).

\begin{tabular}{cccc|ccc}
\hline & \multicolumn{2}{c}{ B2PLYP-D3BJ/jun-cc-pVTZ } & \multicolumn{2}{c}{ DSD-PBEP86-D3BJ/jun-cc-pVTZ } \\
\cline { 2 - 6 } & $\mathrm{X}$ & $\mathrm{Y}$ & $\mathrm{Z}$ & $\mathrm{X}$ & $\mathrm{Y}$ & $\mathrm{Z}$ \\
\hline $\mathrm{S}$ & -0.117089 & -0.000005 & -0.128364 & -0.118394 & -0.000008 & -0.128081 \\
$\mathrm{O}$ & 0.375316 & 0.000014 & 1.261805 & 0.371809 & -0.000023 & 1.259710 \\
$\mathrm{H}$ & 1.914921 & 0.000004 & 1.339109 & 1.907000 & 0.000008 & 1.332815 \\
$\mathrm{C}$ & -1.951846 & 0.000002 & 0.076928 & -1.944745 & 0.000007 & 0.076862 \\
$\mathrm{~F}$ & -2.540749 & -0.000024 & -1.117299 & -2.532275 & 0.000016 & -1.114933 \\
$\mathrm{~F}$ & -2.338948 & -1.083507 & 0.748565 & -2.330200 & -1.081408 & 0.747243 \\
$\mathrm{~F}$ & -2.338946 & 1.083543 & 0.748517 & -2.330180 & 1.081425 & 0.747250 \\
$\mathrm{O}$ & 0.176728 & -1.229372 & -0.849629 & 0.173417 & -1.227179 & -0.848228 \\
$\mathrm{O}$ & 0.176735 & 1.229343 & -0.849662 & 0.173439 & 1.227168 & -0.848210 \\
$\mathrm{O}$ & 2.911670 & 0.000003 & 1.103260 & 2.904171 & 0.000000 & 1.100713 \\
$\mathrm{H}$ & 3.006489 & -0.828492 & 0.512075 & 2.996610 & -0.827335 & 0.507980 \\
$\mathrm{H}$ & 3.006485 & 0.828495 & 0.512069 & 2.996626 & 0.827336 & 0.507989 \\
$\mathrm{O}$ & 2.768936 & 1.993283 & -0.474966 & 2.763156 & 1.990891 & -0.474622 \\
$\mathrm{H}$ & 2.896121 & 2.906454 & -0.205115 & 2.880651 & 2.901792 & -0.192342 \\
$\mathrm{H}$ & 1.822974 & 1.896505 & -0.723152 & 1.817395 & 1.892446 & -0.721940 \\
$\mathrm{O}$ & 2.768936 & -1.993277 & -0.474962 & 2.763139 & -1.990886 & -0.474623 \\
$\mathrm{H}$ & 1.822973 & -1.896511 & -0.723143 & 1.817378 & -1.892443 & -0.721945 \\
$\mathrm{H}$ & 2.896143 & -2.906454 & -0.205139 & 2.880623 & -2.901774 & -0.192296 \\
\hline
\end{tabular}

B3LYP-D3BJ/jun-cc-pVDZ

\begin{tabular}{cccc|ccc} 
& \multicolumn{5}{c}{ B3LYP-D3BJ/jun-cc-pVDZ } \\
\cline { 2 - 7 } & $\mathrm{X}$ & Equilibrium & $\mathrm{Y}$ & $\mathrm{C}$ Ground vibrational state \\
\hline $\mathrm{S}$ & 0.116135 & 0.000005 & -0.136450 & 0.114874 & 0.000004 & -0.141924 \\
$\mathrm{O}$ & -0.391459 & 0.000003 & 1.267266 & -0.400202 & 0.000002 & 1.263879 \\
$\mathrm{H}$ & -1.932489 & -0.000014 & 1.364157 & -1.927413 & -0.000014 & 1.364293 \\
$\mathrm{C}$ & 1.966712 & -0.000003 & 0.081964 & 1.972574 & -0.000004 & 0.083674 \\
$\mathrm{~F}$ & 2.566890 & -0.000007 & -1.115207 & 2.576070 & -0.000007 & -1.114137 \\
$\mathrm{~F}$ & 2.351738 & 1.089742 & 0.759890 & 2.357683 & 1.090876 & 0.762780 \\
$\mathrm{~F}$ & 2.351728 & -1.089752 & 0.759890 & 2.357678 & -1.090886 & 0.762781 \\
$\mathrm{O}$ & -0.180320 & 1.243598 & -0.865777 & -0.179863 & 1.245685 & -0.871975 \\
$\mathrm{O}$ & -0.180330 & -1.243577 & -0.865791 & -0.179877 & -1.245664 & -0.871989 \\
$\mathrm{O}$ & -2.936639 & -0.000005 & 1.129118 & -2.947635 & -0.000006 & 1.130435 \\
$\mathrm{H}$ & -3.021843 & 0.831030 & 0.524809 & -3.037532 & 0.841411 & 0.523880 \\
$\mathrm{H}$ & -3.021854 & -0.831035 & 0.524804 & -3.037543 & -0.841422 & 0.523874 \\
$\mathrm{O}$ & -2.776194 & -1.970024 & -0.478238 & -2.777005 & -1.984158 & -0.467381 \\
$\mathrm{H}$ & -2.931583 & -2.904785 & -0.280183 & -2.962342 & -2.891868 & -0.325240 \\
$\mathrm{H}$ & -1.825699 & -1.889601 & -0.749195 & -1.833010 & -1.909247 & -0.748699 \\
$\mathrm{O}$ & -2.776196 & 1.970021 & -0.478238 & -2.777008 & 1.984154 & -0.467382 \\
$\mathrm{H}$ & -1.825689 & 1.889585 & -0.749168 & -1.832996 & 1.909238 & -0.748690 \\
$\mathrm{H}$ & -2.931568 & 2.904781 & -0.280165 & -2.962349 & 2.891927 & -0.325221 \\
\hline
\end{tabular}


Table S26. Cartesian coordinates (in $\AA$ ) of the minimum-energy structure of $\mathrm{CF}_{3} \mathrm{SO}_{3} \mathrm{H} \cdots\left(\mathrm{H}_{2} \mathrm{O}\right)_{3}$ structure (IIIB) resulting from B2PLYP-D3BJ/jun-cc-pVTZ and DSD-PBEP86-D3BJ/jun-cc-pVTZ calculations (top). Cartesian coordinates of the equilibrium and vibrationally averaged structure calculated at the B3LYP-D3BJ/jun-cc-pVDZ level (bottom).

\begin{tabular}{cccc|ccc}
\hline & \multicolumn{2}{c}{ B2PLYP-D3BJ/jun-cc-pVTZ } & \multicolumn{2}{c}{ DSD-PBEP86-D3BJ/jun-cc-pVTZ } \\
\cline { 2 - 6 } & $\mathrm{X}$ & $\mathrm{Y}$ & $\mathrm{Z}$ & $\mathrm{X}$ & $\mathrm{Y}$ & $\mathrm{Z}$ \\
\hline $\mathrm{S}$ & -0.116617 & -0.021727 & -0.129852 & -0.118023 & -0.026212 & -0.129174 \\
$\mathrm{O}$ & 0.379434 & 0.012508 & 1.257950 & 0.375614 & 0.016460 & 1.256092 \\
$\mathrm{H}$ & 1.918205 & 0.007264 & 1.346215 & 1.909946 & 0.010963 & 1.341249 \\
$\mathrm{C}$ & -1.950860 & 0.009438 & 0.078086 & -1.943793 & 0.011738 & 0.077758 \\
$\mathrm{~F}$ & -2.541042 & -0.017371 & -1.115513 & -2.532429 & -0.021410 & -1.113326 \\
$\mathrm{~F}$ & -2.351968 & -1.047196 & 0.781795 & -2.346602 & -1.036750 & 0.787350 \\
$\mathrm{~F}$ & -2.321098 & 1.118611 & 0.716887 & -2.309163 & 1.124252 & 0.707660 \\
$\mathrm{O}$ & 0.160069 & -1.273702 & -0.817539 & 0.153060 & -1.281591 & -0.806798 \\
$\mathrm{O}$ & 0.191202 & 1.183528 & -0.887360 & 0.191518 & 1.170658 & -0.893906 \\
$\mathrm{O}$ & 2.915521 & 0.021863 & 1.110964 & 2.907801 & 0.025630 & 1.110352 \\
$\mathrm{H}$ & 3.020785 & -0.794536 & 0.505951 & 3.011211 & -0.789540 & 0.503870 \\
$\mathrm{H}$ & 2.995463 & 0.853659 & 0.524786 & 2.985061 & 0.855695 & 0.521832 \\
$\mathrm{O}$ & 2.751786 & 2.002466 & -0.486936 & 2.744642 & 2.000303 & -0.488787 \\
$\mathrm{H}$ & 2.858899 & 2.921379 & -0.227865 & 2.838820 & 2.918185 & -0.220564 \\
$\mathrm{H}$ & 1.807782 & 1.882572 & -0.736025 & 1.801864 & 1.874848 & -0.738776 \\
$\mathrm{O}$ & 2.754257 & -2.022394 & -0.402282 & 2.750788 & -2.015907 & -0.401173 \\
$\mathrm{H}$ & 1.812404 & -1.926680 & -0.665570 & 1.807090 & -1.924569 & -0.658457 \\
$\mathrm{H}$ & 3.253778 & -2.183650 & -1.206804 & 3.243127 & -2.159450 & -1.213681 \\
\hline
\end{tabular}

B3LYP-D3BJ/jun-cc-pVDZ

\begin{tabular}{|c|c|c|c|c|c|c|}
\hline & \multicolumn{6}{|c|}{ B3LYP-D3BJ/jun-cc-pVDZ } \\
\hline & \multicolumn{3}{|c|}{ Equilibrium } & \multicolumn{3}{|c|}{ Ground vibrational state } \\
\hline & $\mathrm{X}$ & $\mathrm{Y}$ & Z & $X$ & $\mathrm{Y}$ & Z \\
\hline$S$ & -0.116010 & -0.025965 & -0.136996 & -0.114303 & -0.019300 & -0.142241 \\
\hline $\mathrm{O}$ & 0.395094 & 0.014136 & 1.264014 & 0.402827 & 0.017047 & 1.261674 \\
\hline $\mathrm{H}$ & 1.936632 & 0.008663 & 1.375329 & 1.928312 & 0.011412 & 1.369697 \\
\hline $\mathrm{C}$ & -1.966125 & 0.011811 & 0.082800 & -1.971130 & 0.009860 & 0.084452 \\
\hline $\mathrm{F}$ & -2.566795 & -0.019874 & -1.114001 & -2.575119 & -0.017147 & -1.113152 \\
\hline $\mathrm{F}$ & -2.368764 & -1.045634 & 0.798630 & -2.368550 & -1.055288 & 0.794760 \\
\hline $\mathrm{F}$ & -2.331332 & 1.132062 & 0.721210 & -2.341419 & 1.125567 & 0.731145 \\
\hline $\mathrm{O}$ & 0.160522 & -1.295952 & -0.826809 & 0.166388 & -1.287273 & -0.837739 \\
\hline $\mathrm{O}$ & 0.197724 & 1.189099 & -0.908741 & 0.192449 & 1.202537 & -0.908994 \\
\hline $\mathrm{O}$ & 2.941007 & 0.025026 & 1.141245 & 2.949160 & 0.025734 & 1.136352 \\
\hline $\mathrm{H}$ & 3.036935 & -0.792699 & 0.521530 & 3.051251 & -0.809903 & 0.526611 \\
\hline $\mathrm{H}$ & 3.009382 & 0.858378 & 0.540578 & 3.023484 & 0.864848 & 0.526454 \\
\hline $\mathrm{O}$ & 2.760501 & 1.974241 & -0.494490 & 2.762164 & 1.986268 & -0.493944 \\
\hline $\mathrm{H}$ & 2.892011 & 2.915157 & -0.308057 & 2.922481 & 2.899524 & -0.345946 \\
\hline $\mathrm{H}$ & 1.811712 & 1.867164 & -0.766453 & 1.817523 & 1.888117 & -0.772441 \\
\hline $\mathrm{O}$ & 2.764545 & -1.989381 & -0.412058 & 2.764159 & -2.013374 & -0.394854 \\
\hline $\mathrm{H}$ & 1.810718 & -1.927205 & -0.675721 & 1.819155 & -1.956512 & -0.674553 \\
\hline $\mathrm{H}$ & 3.262129 & -2.186378 & -1.218693 & 3.264025 & -2.308136 & -1.134346 \\
\hline
\end{tabular}


Table S27. Cartesian coordinates (in $\AA$ ) of the minimum-energy structure of $\mathrm{CF}_{3} \mathrm{SO}_{3} \mathrm{H} \cdots\left(\mathrm{H}_{2} \mathrm{O}\right)_{3}$ structure (IIIC) resulting from B2PLYP-D3BJ/jun-cc-pVTZ and DSD-PBEP86-D3BJ/jun-cc-pVTZ calculations (top). Cartesian coordinates of the equilibrium and vibrationally averaged structure calculated at the B3LYP-D3BJ/jun-cc-pVDZ level (bottom).

\begin{tabular}{cccc|ccc}
\hline & \multicolumn{3}{c|}{ B2PLYP-D3BJ/jun-cc-pVTZ } & \multicolumn{2}{c}{ DSD-PBEP86-D3BJ/jun-cc-pVTZ } \\
\cline { 2 - 6 } & $\mathrm{X}$ & $\mathrm{Y}$ & $\mathrm{Z}$ & $\mathrm{X}$ & $\mathrm{Y}$ & $\mathrm{Z}$ \\
\hline $\mathrm{S}$ & -0.855705 & 0.630656 & 0.648460 & -0.872468 & 0.631270 & 0.633164 \\
$\mathrm{O}$ & -0.413419 & 1.794913 & -0.242971 & -0.451577 & 1.796353 & -0.260570 \\
$\mathrm{H}$ & 0.624034 & 1.833537 & -0.430972 & 0.589256 & 1.849389 & -0.429880 \\
$\mathrm{C}$ & -1.067467 & -0.711185 & -0.608544 & -1.017096 & -0.722635 & -0.607406 \\
$\mathrm{~F}$ & -2.009917 & -0.390427 & -1.485991 & -1.954186 & -0.444017 & -1.500749 \\
$\mathrm{~F}$ & 0.085857 & -0.899053 & -1.260529 & 0.150457 & -0.874341 & -1.237682 \\
$\mathrm{~F}$ & -1.403184 & -1.842832 & 0.004113 & -1.321242 & -1.857352 & 0.010152 \\
$\mathrm{O}$ & -2.144516 & 0.891881 & 1.194077 & -2.176349 & 0.855649 & 1.152691 \\
$\mathrm{O}$ & 0.225680 & 0.185516 & 1.492601 & 0.206883 & 0.229038 & 1.497281 \\
$\mathrm{O}$ & 2.003976 & 1.970936 & -0.774942 & 1.969331 & 1.997273 & -0.741918 \\
$\mathrm{H}$ & 2.474620 & 2.638478 & -0.268964 & 2.425232 & 2.644236 & -0.196715 \\
$\mathrm{H}$ & 2.552257 & 1.140936 & -0.737273 & 2.512661 & 1.164478 & -0.705484 \\
$\mathrm{O}$ & 3.457522 & -0.222187 & -0.629480 & 3.417177 & -0.199498 & -0.602701 \\
$\mathrm{H}$ & 3.541607 & -0.758374 & -1.421615 & 3.500539 & -0.727877 & -1.400341 \\
$\mathrm{H}$ & 3.122286 & -0.820962 & 0.073737 & 3.074442 & -0.806953 & 0.088495 \\
$\mathrm{O}$ & 2.292584 & -1.689225 & 1.321248 & 2.232271 & -1.692773 & 1.319427 \\
$\mathrm{H}$ & 1.525310 & -1.131863 & 1.529501 & 1.478542 & -1.122498 & 1.540866 \\
$\mathrm{H}$ & 2.689464 & -1.924060 & 2.163265 & 2.624422 & -1.948437 & 2.157754 \\
\hline
\end{tabular}

B3LYP-D3BJ/jun-cc-pVDZ

\begin{tabular}{cccc|ccc} 
& \multicolumn{5}{c}{ B3LYP-D3BJ/jun-cc-pVDZ } \\
\cline { 2 - 7 } & $\mathrm{X}$ & Equilibrium & $\mathrm{Z}$ & \multicolumn{3}{c}{ Ground vibrational state } \\
\hline $\mathrm{S}$ & -0.853822 & 0.650443 & 0.643868 & -0.843919 & 0.657684 & 0.634921 \\
$\mathrm{O}$ & -0.412732 & 1.812211 & -0.283286 & -0.378284 & 1.794343 & -0.299899 \\
$\mathrm{H}$ & 0.644647 & 1.840418 & -0.481099 & 0.735660 & 1.853490 & -0.486659 \\
$\mathrm{C}$ & -1.069719 & -0.730437 & -0.598701 & -1.072881 & -0.741835 & -0.595047 \\
$\mathrm{~F}$ & -2.037608 & -0.440664 & -1.470240 & -2.028105 & -0.450484 & -1.483163 \\
$\mathrm{~F}$ & 0.082144 & -0.913157 & -1.272741 & 0.084530 & -0.954657 & -1.259537 \\
$\mathrm{~F}$ & -1.376438 & -1.864807 & 0.041429 & -1.403477 & -1.868249 & 0.052616 \\
$\mathrm{O}$ & -2.151984 & 0.919350 & 1.199371 & -2.143319 & 0.934830 & 1.192156 \\
$\mathrm{O}$ & 0.248746 & 0.217270 & 1.496004 & 0.248122 & 0.209351 & 1.501573 \\
$\mathrm{O}$ & 1.999907 & 1.946372 & -0.816766 & 2.011146 & 1.982419 & -0.760651 \\
$\mathrm{H}$ & 2.492761 & 2.616483 & -0.321013 & 2.460661 & 2.613983 & -0.230140 \\
$\mathrm{H}$ & 2.549533 & 1.101793 & -0.777279 & 2.554333 & 1.121918 & -0.749353 \\
$\mathrm{O}$ & 3.432220 & -0.240034 & -0.640548 & 3.370829 & -0.222147 & -0.672265 \\
$\mathrm{H}$ & 3.526543 & -0.817973 & -1.411265 & 3.453102 & -0.761940 & -1.431953 \\
$\mathrm{H}$ & 3.122125 & -0.825075 & 0.099519 & 3.086665 & -0.815007 & 0.068928 \\
$\mathrm{O}$ & 2.30834 & -1.643279 & 1.360326 & 2.320254 & -1.630145 & 1.354934 \\
$\mathrm{H}$ & 1.524706 & -1.090015 & 1.560055 & 1.528946 & -1.109697 & 1.562807 \\
$\mathrm{H}$ & 2.681813 & -1.889859 & 2.218089 & 2.664331 & -1.929484 & 2.165657 \\
\hline
\end{tabular}


Table S28. Cartesian coordinates (in $\AA$ ) of the minimum-energy structure of $\mathrm{CF}_{3} \mathrm{SO}_{3} \mathrm{H} \cdots\left(\mathrm{H}_{2} \mathrm{O}\right)_{3}$ structure (IIID) resulting from B2PLYP-D3BJ/jun-cc-pVTZ and DSD-PBEP86-D3BJ/jun-cc-pVTZ calculations (top). Cartesian coordinates of the equilibrium and vibrationally averaged structure calculated at the B3LYP-D3BJ/jun-cc-pVDZ level (bottom).

\begin{tabular}{cccc|ccc}
\hline & \multicolumn{2}{c|}{ B2PLYP-D3BJ/jun-cc-pVTZ } & \multicolumn{2}{c}{ DSD-PBEP86-D3BJ/jun-cc-pVTZ } \\
\cline { 2 - 6 } & $\mathrm{X}$ & $\mathrm{Y}$ & $\mathrm{Z}$ & $\mathrm{X}$ & $\mathrm{Y}$ & $\mathrm{Z}$ \\
\hline $\mathrm{S}$ & -0.159763 & -0.146696 & -0.041321 & -0.157252 & 0.155725 & 0.026852 \\
$\mathrm{O}$ & 0.294699 & 0.700090 & 1.089971 & 0.289821 & -0.693817 & -1.100088 \\
$\mathrm{H}$ & 1.297872 & 1.596436 & 0.566737 & 1.285982 & -1.601083 & -0.566023 \\
$\mathrm{C}$ & -1.998455 & 0.016495 & 0.025194 & -1.986808 & -0.020830 & -0.017548 \\
$\mathrm{~F}$ & -2.461963 & -0.423521 & 1.192664 & -2.464798 & 0.407152 & -1.180449 \\
$\mathrm{~F}$ & -2.347047 & 1.295680 & -0.121929 & -2.322545 & -1.299199 & 0.141305 \\
$\mathrm{~F}$ & -2.551220 & -0.694533 & -0.955805 & -2.532714 & 0.690910 & 0.962956 \\
$\mathrm{O}$ & 0.090011 & -1.560386 & 0.151977 & 0.078968 & 1.567397 & -0.176221 \\
$\mathrm{O}$ & 0.233461 & 0.411941 & -1.326661 & 0.250015 & -0.389993 & 1.311379 \\
$\mathrm{O}$ & 2.020906 & 2.054078 & -0.053301 & 1.997150 & -2.054672 & 0.063748 \\
$\mathrm{H}$ & 1.618992 & 1.874784 & -0.927276 & 1.587505 & -1.844502 & 0.928540 \\
$\mathrm{H}$ & 2.856247 & 1.429166 & 0.013402 & 2.834348 & -1.433205 & -0.007718 \\
$\mathrm{O}$ & 3.912317 & 0.424016 & 0.076315 & 3.892404 & -0.435520 & -0.073982 \\
$\mathrm{H}$ & 4.522075 & 0.460132 & 0.817948 & 4.489801 & -0.468915 & -0.825948 \\
$\mathrm{H}$ & 3.544487 & -0.501752 & 0.044524 & 3.525913 & 0.490075 & -0.038826 \\
$\mathrm{O}$ & 2.831429 & -1.982996 & -0.001044 & 2.822211 & 1.974701 & 0.013084 \\
$\mathrm{H}$ & 3.000603 & -2.537651 & -0.766927 & 2.977942 & 2.510477 & 0.795324 \\
$\mathrm{H}$ & 1.857968 & -1.954033 & 0.091700 & 1.850437 & 1.952966 & -0.093824 \\
\hline
\end{tabular}

\begin{tabular}{cccc|ccc}
\hline & \multicolumn{5}{c}{ B3LYP-D3BJ/jun-cc-pVDZ } \\
\cline { 2 - 7 } & $\mathrm{X}$ & Equilibrium & $\mathrm{Z}$ & $\mathrm{X}$ & $\mathrm{Y}$ & $\mathrm{Z}$ \\
\hline $\mathrm{S}$ & -0.150026 & 0.153546 & 0.029121 & -0.174808 & 0.175764 & 0.200613 \\
$\mathrm{O}$ & 0.301793 & -0.739836 & -1.092913 & 0.345259 & -0.808163 & -0.873634 \\
$\mathrm{H}$ & 1.291446 & -1.629391 & -0.557293 & 1.106822 & -1.424061 & -0.513872 \\
$\mathrm{C}$ & -2.005927 & -0.016945 & -0.017748 & -2.033686 & -0.005228 & -0.050196 \\
$\mathrm{~F}$ & -2.485965 & 0.389516 & -1.199013 & -2.388604 & 0.316405 & -1.312546 \\
$\mathrm{~F}$ & -2.348934 & -1.300736 & 0.170702 & -2.399838 & -1.275451 & 0.196439 \\
$\mathrm{~F}$ & -2.556659 & 0.723243 & 0.953486 & -2.685257 & 0.808039 & 0.811179 \\
$\mathrm{O}$ & 0.100348 & 1.575788 & -0.212713 & 0.101034 & 1.568200 & -0.186610 \\
$\mathrm{O}$ & 0.265990 & -0.375903 & 1.336865 & 0.148585 & -0.279517 & 1.535919 \\
$\mathrm{O}$ & 2.030443 & -2.088707 & 0.062814 & 2.164209 & -2.225776 & -0.253029 \\
$\mathrm{H}$ & 1.636191 & -1.904678 & 0.945405 & 2.120665 & -2.731229 & 0.865849 \\
$\mathrm{H}$ & 2.858821 & -1.436340 & -0.011137 & 2.937042 & -1.505028 & -0.221866 \\
$\mathrm{O}$ & 3.882287 & -0.415188 & -0.081933 & 3.933614 & -0.390527 & -0.212577 \\
$\mathrm{H}$ & 4.516031 & -0.431832 & -0.814051 & 4.603470 & -0.355235 & -0.875749 \\
$\mathrm{H}$ & 3.522402 & 0.524998 & -0.032249 & 3.566798 & 0.541349 & -0.115345 \\
$\mathrm{O}$ & 2.829036 & 1.994247 & 0.018963 & 2.849709 & 2.024191 & 0.00417 \\
$\mathrm{H}$ & 2.961985 & 2.552602 & 0.798992 & 2.984374 & 2.561052 & 0.753862 \\
$\mathrm{H}$ & 1.852328 & 1.986015 & -0.124948 & 1.882095 & 2.017134 & -0.137252 \\
\hline
\end{tabular}

